Cipango
Cipango

$22 \mid 2015$

Du particulier et de l'universel

\title{
Portrait socio-économique des galeries japonaises
}

Stratégies de développement et construction de la valeur artistique

Strategies of Development and Construction of Artistic Value

A Socio-Economic Analysis of Japanese Galleries

\section{Cléa Patin}

\section{(2) OpenEdition}

\section{Journals}

Édition électronique

URL : https://journals.openedition.org/cipango/3222

DOI : $10.4000 /$ cipango.3222

ISSN : 2260-7706

Éditeur

INALCO

Édition imprimée

Date de publication : 1 janvier 2015

ISSN : 1164-5857

Référence électronique

Cléa Patin, « Portrait socio-économique des galeries japonaises », Cipango [En ligne], 22 | 2015, mis en ligne le 13 février 2019, consulté le 09 avril 2022. URL : http://journals.openedition.org/cipango/3222 ; DOI : https://doi.org/10.4000/cipango.3222

\section{(c) (7) \&}

Cipango est mis à disposition selon les termes de la Licence Creative Commons Attribution - Pas d'Utilisation Commerciale 4.0 International. 


\section{Portrait socio-économique des galeries japonaises}

\section{Stratégies de développement et construction de la valeur artistique}

Strategies of Development and Construction of Artistic Value A Socio-Economic Analysis of Japanese Galleries

Cléa Patin

Maître de conférences, université Jean Moulin Lyon 3

Les artistes, prompts à développer un discours misérabiliste, se plaignent inlassablement : « Au Japon, il n’y a pas de galeries ! ». À les croire, le pays ne pourrait compter sur des marchands d'art capables de s'engager auprès de ceux en voie de reconnaissance ou de soutenir la production des œuvres en amont ${ }^{1}$. Certes, la complexité des réseaux marchands ne facilite pas la lisibilité à l'extérieur, mais il faut nuancer ces jugements pour le moins sévères. Quels sont donc les atouts des galeristes au Japon pour produire les artistes vivants, par rapport aux autres acteurs du marché ? Comment se nouent leurs relations avec les artistes et les

1. Pour des citations d'artistes, tirées d'entretiens ou de revues d'art, nous renvoyons au chapitre 4 de notre thèse, Une Approche sociologique du marché de l'art au Japon (EHESS, 2012), p. 276-296. 
260 Cahiers d'études japonaises n²2

collectionneurs, au niveau du choix des œuvres et de la gestion des transactions ? Ont-ils les moyens de promouvoir leurs poulains sur le segment le plus globalisé et, plus modestement, de viabiliser leur activité ?

De fait, contrairement aux assertions des artistes, il existe des galeries au Japon, qui jouent un rôle central autant qu'incontournable. En effet, des établissements se chargent d'exhumer les nouvelles tendances - auquel cas ils opèrent alors dans un contexte d'incertitude à partir d'un excès structurel d'offre - et se mettent en rapport avec des collectionneurs, des musées, des acheteurs, souvent en concurrence avec des maisons de vente aux enchères. Par la formation de la demande et la sélection au sein de l'offre, ils contrôlent une partie du mécanisme de construction des valeurs artistiques. Or, pour comprendre s'ils parviennent à relever les défis qu'implique ce rôle, il faut d'abord saisir leur place globale sur le marché de l'art (perspective macro), ainsi que leur fonctionnement sur le terrain (travail d'enquête), avant d'évaluer les risques de fragilisation qui les menacent à terme, du fait de mécanismes de prédation exacerbés ${ }^{2}$.

2. Nous nous situons ici dans la filiation des travaux pionniers de Raymonde Moulin sur le marché de l'art en France, Marcya Bystrin et Katherine Guiffre sur les interactions entre galeries aux États-Unis et enfin Françoise Benhamou, Nathalie Moureau et Dominique Sagot-Duvauroux sur les stratégies économiques des galeries parisiennes. Voir Raymond Moulin, Le Marché de la peinture en France, Paris, Les Éditions de Minuit, 1967 (réed. 1992) ; Marcia N. Bystryn, "Variation in Artistic Circles", The Sociological Quarterly, vol. 22, no 1, 1981, pp. 119-132 ; Katherine Giuffre, "Sandpiles of Opportunity: Success in the Art World", Social Forces, vol. 77, n 3, Chapel Hill, University of North Carolina Press, Mar. 1999, pp. 815-832; Françoise Benhamou, Nathalie Moureau, Dominique Sagot-Duvauroux, Les Galeries d'art contemporain en France. Portraits et enjeux dans un marché mondialisé, Paris, La Documentation française, 2001. 


\section{La place globale des galeries sur le marché de l'art : un milieu polarisé, mais fragile}

\section{Deux types de galeries}

Au Japon, les galeries se distinguent classiquement en deux grands groupes, selon qu'il s'agit d'établissements de négoce ou de promotion ${ }^{3}$. Les premières se spécialisent sur le segment de l'art classé, à la qualité reconnue et établie, et acquièrent des œuvres pour les revendre avec une plus-value. Elles agissent donc principalement sur le second marché, à savoir celui des reventes. Au Japon, ces galeries puisent leurs racines dans le commerce lié à la cérémonie du thé, en droite lignée des karamonoya 唐物屋 ${ }^{4}$. Encore très actives au sein d'associations professionnelles comme le Tōkyō Art Club, elles s'imposent toujours comme l'intermédiaire privilégié sur le marché de l'art moderne et ancien. Cependant, les frontières ne sont pas étanches : certaines agissent aussi dans le domaine de l'art contemporain.

Lancer des artistes à forte créativité revient à expérimenter un déficit permanent. [...] Il faut gagner de l'argent à côté. Par exemple, on peut s'assurer une assise stable avec des artistes déjà cotés. Ensuite, quand on entend que tel collectionneur souhaite se défaire d'une œuvre et que l'on connaît un acheteur potentiel, on peut jouer le rôle d'entremetteur, en prenant cette œuvre en dépôt. (Galerie Mushanokōji ギャラリー武者小路)

3. Raymonde Moulin, L'Artiste, l'institution et le marché, Paris, Champs Flammarion, 1992, p. $45-51$.

4. Tōkyō bijutsu kurabu 東京美術俱楽部, Tōkyō bijutsu shijō-shi 東京美術市場史 (Histoire du marché de l'art tokyoïte), Tōkyō, Tōkyō bijutsu kurabu, 1979, p. 17-63; Tōkyō bijutsu kurabu hyakunen-shi hensan iin-kai 東京美術俱楽部百年史編纂委員 会, Bijutsushō no hyakunen : Tökyō bijutsu kurabu hyakunen-shi 美術商の百年：東京 美術俱楽部百年史 (Le siècle des marchands d'art : 100 ans d'histoire du Club des Arts de Tōkyō), Tōkyō, Tōkyō Bijutsu kurabu/Tōkyō bijutsu-shō kyōdō kumiai 東京美術俱 楽部・東京美術商協同組合, 2006, p. 59-138. 
Les premiers galeristes à s'être tournés vers l'art contemporain au tout début des années 1970 (Tōkyō garō, Minami-garō) étaient issus de l'art ancien. Ils correspondaient à ce qu'on appelle des « experts ». Un jour, ils ont voulu essayer autre chose. Mais l'art contemporain ne se vendait pas du tout : il n'y avait pas encore de marché. Alors en coulisses, ils ont continué à vendre de l'art ancien. (Galerie Soh 双ギャラリー)

Il était impossible pour une galerie de ne vivre que de photographies... Moi, j'ai pu m'en sortir parce que j'avais les tableaux - William Bouguereau, Vieira Da Silva, etc. -, qui ont pris de la valeur. Quand j'avais des rentrées sur ce secteur, je les réinjectais du côté des jeunes photographes. (Galerie Zeit Foto ツ アイト・フォト)

En comparaison, les galeries de promotion, sur le modèle de Durand-Ruel, Vollard, ou Kahnweiler, sont apparues au cours des années 1910 et surtout 1920. Qu'il s'agisse de structures fonctionnant un peu comme des foyers d'artistes, telles Rōkandō 琅かん洞 5 , Kabutoya gadō 兒屋画堂 ou Venus kurabu ヴィナス俱 楽部 ${ }^{6}$, ou des premiers marchands de peinture à l'huile, comme la galerie Nichidō 日動画廊 ${ }^{7}$, celles-ci privilégient la production active des peintres de leur temps, pour lesquels elles se doivent de créer une demande. Aujourd'hui encore, elles constituent le socle du premier marché - celui sur lequel les œuvres sont mises en circulation pour la première fois.

5. NAKANISHI Takaki 中西たかき, 《Kotarō to Nihon hatsu no garō » 小太郎と日本 初の画廊 (Kotarō et la première galerie japonaise), KANDAルネッサンス (KANDA Renaissance), ${ }^{\circ} 18,20$ juillet 1991, p. 10-12.

6. Michael LuCKen, L'Art du Japon au vingtième siècle : pensée, formes, résistances, Paris, Hermann, 2001, p. 45.

7. Nihon yōgashō kyōdō kumiai 日本洋画商協同組合, Nihon yōgashō-shi 日本洋画 商史 (Histoire des marchands d'art occidental), Tōkyō, Bijutsu shuppan-sha 美術出版 社, 1985, p. 279-389. 
Le lancement d'un artiste suppose un gros investissement sur le plan financier. Les frais sont très importants. Il n'est même pas pensable de faire un bénéfice avant la troisième ou la quatrième exposition. Pendant ce laps de temps où l'on s'investit totalement, l'artiste doit rester fidèle. Après, une fois que l'on commence à vendre, c'est différent. (Galerie Shirota 白田画廊)

Toutefois, de même que les galeries de négoce s'immiscent sur le premier marché, les galeries de promotion arrondissent leurs fins de mois en tant qu'intermédiaires sur le marché secondaire, moyennant une commission.

Nous présentons en priorité les ouvres d'artistes jeunes, spécialisés dans l'art contemporain. Mais une galerie ne peut survivre uniquement avec ça... Alors nous jouons aussi le rôle d'intermédiaires entre des clients qui souhaitent se défaire de tableaux et d'autres qui cherchent à en acquérir. Ces ouvres n’apparaissent pas dans nos expositions. (Galerie Marunouchi 丸 の内ギャラリー)

Ensuite, surtout au Japon, on distingue clairement les galeries programmatrices des galeries locatrices. En effet, si tous les établissements ont pour but d'exposer pour vendre des œuvres, leurs moyens differrent : les galeries « programmatrices » (kikaku garō 企画画廊) financent elles-mêmes leurs expositions, tandis que les galeries locatrices (kashi garō 貸画廊) se déchargent d'une partie des coûts sur les artistes, par la perception d'un loyer. Nées dans les années 1930 à l'initiative des librairies Kinokuniya 紀伊國屋 et Zanmaidō三昧堂, leur sélection esthétique apparaît moins rigoureuse que celle de leurs rivales, mais participe aussi à la diffusion de l'offre : à travers elles, n'importe quel artiste débutant s'est vu octroyer la possibilité d'exposer, dans l'espoir de s'attirer les bonnes grâces de la critique.

Entre ces deux pôles, il existe cependant toute une série de degrés. Viennent d'abord les galeries en « programmation pure », souvent bien implantées tant sur le premier marché (celui accueillant les œuvres mises en vente pour la première fois), que sur le second marché (celui des reventes). Elles sont suivies par les galeries « à proportion de location », qui compensent les pertes occasionnées lors du lancement de leurs poulains en louant leur espace à des artistes de passage. 
Toutefois, leur idéal reste d'agir sur le premier marché : dès que leur budget le permet, elles augmentent la proportion de programmation. Enfin, viennent les galeries purement locatrices, qui ne sont pas des intermédiaires actifs. Sortes $\mathrm{d}$ '《agents immobiliers » du marché de l'art, elles ne mènent pas véritablement d'action de promotion. Quoi qu'il en soit, l'idéal de programmation pure demeure le stade ultime de distinction et de reconnaissance au sein des galeristes :

Acquérir des œuvres auprès des artistes et les prendre en dépôt constitue l'apanage des galeries locatrices. Cela les différencie fondamentalement des galeries locatrices, qui s'apparentent plutôt à des agents immobiliers. Les galeries programmatrices sont les seules vraies galeries. En effet, l'achat d'œuvres met en avant notre propre responsabilité : nous faisons acte de confiance en nous-mêmes et dans les artistes. (Galerie Kotōken 古陶軒)

Il arrive que les deux systèmes (location et programmation) coexistent au sein d'une même galerie. Mais dans ce cas, cela se gère dans deux espaces distincts. Évidemment, les artistes en location n'apprécient guère de subventionner leurs camarades en programmation pure. (Collectionneur S)

La plupart des directeurs de galeries locatrices sont des propriétaires fonciers. Ils s'engagent sur le marché de l'art de manière provisoire, pendant quelques mois voire plusieurs années, en attendant de trouver un acheteur pour leurs locaux ou une entreprise susceptible de les louer - auquel cas ils ferment. Ce ne sont pas de vrais professionnels. (Galerie Shinobazu 不忍ギャラ リー)

Comment se répartit le nombre de galeries pour chacun de ces types ? Pour l'année fiscale 2006-2007, l'annuaire Bijutsu nenkan 美術年鑑 recense 
696 galeries en programmation pure (39,3\%), 301 en semi-programmation (17\%) et 119 en location $(6,7 \%)^{8}$.

Or, ces caractéristiques par types se trouvent étroitement corrélées aux choix esthétiques, aux modes de promotion et surtout à la force de frappe sur le plan financier.

\section{Des revenus instables, quoique parfois importants}

Trouver des estimations globales sur la force financière des galeries constitue un point épineux : d'abord, parce qu'il s'agit d'une information cruciale, que les intéressés répugnent à transmettre ; ensuite, parce qu'à partir de 2005, le Bureau des Impôts a cessé de rendre public les déclarations fiscales des entreprises dont le revenu annuel dépasse 40 millions de yens (280 000 euros). Voici toutefois quelques données fournies par Segi Shin.ichi, concernant le chiffre d'affaires, puis la part des importations.

TAB. 1 : GALERIES PRÉSENTES DANS LE TOP TRENTE DES ANNÉES

FISCALES 2000 ET 2005

\begin{tabular}{|c|c|c|c|c|c|c|c|c|c|}
\hline \multicolumn{2}{|c|}{ Nom de la galerie } & \multicolumn{4}{|c|}{$\begin{array}{c}\text { Chiffre d'affaires } \\
\text { (en millions } ¥)\end{array}$} & \multicolumn{4}{|c|}{$\begin{array}{c}\text { Revenus déclarés } \\
\text { (en millions } ¥)\end{array}$} \\
\hline & & & 2000 & & 2005 & & 2000 & & 2005 \\
\hline $\begin{array}{c}\text { Art } \\
\text { vivant }\end{array}$ & $\begin{array}{l}\text { アール } \\
\text { ビバン }\end{array}$ & 1 & 13597 & 1 & 6909 & 27 & 4,8 & 1 & 1175 \\
\hline $\begin{array}{c}\text { Art } \\
\text { collection } \\
\text { house }\end{array}$ & $\begin{array}{c}\text { アート } \\
\text { コレクション } \\
\text { ハウス }\end{array}$ & 2 & 8007 & 2 & 4750 & 11 & 74 & 15 & 67 \\
\hline $\begin{array}{c}\text { Art } \\
\text { brillant }\end{array}$ & $\begin{array}{l}\text { アール } \\
\text { ブリアン }\end{array}$ & 4 & 5119 & 4 & 2175 & 1 & 739 & 3 & 225 \\
\hline $\begin{array}{c}\text { Art } \\
\text { Yomiuri }\end{array}$ & $\begin{array}{l}\text { アート } \\
\text { よみうり }\end{array}$ & 13 & 1500 & 14 & 600 & 13 & 65 & 10 & 80 \\
\hline
\end{tabular}

8. Le reste des galeries ne précise pas son type, mais nous pensons qu'il s'agit surtout de galeries locatrices, qui ont plus à gagner en prestige en laissant planer un flou. 


\begin{tabular}{|c|c|c|c|c|c|c|c|c|c|}
\hline $\begin{array}{c}\text { Kaitakudō } \\
\text { art }\end{array}$ & $\begin{array}{l}\text { 偕拓堂 } \\
\text { アート }\end{array}$ & 16 & 1210 & 6 & 1480 & 15 & 61 & 19 & 61 \\
\hline Kochūkyo & 壷中居 & 18 & 1000 & 5 & 1600 & 26 & 40 & 4 & 167 \\
\hline $\begin{array}{c}\text { Maruyama } \\
\text { and Co. }\end{array}$ & 細山龍泉堂 & 20 & 700 & 9 & 905 & 22 & 47 & 17 & 66 \\
\hline
\end{tabular}

Source : Segi Shin.ichi 瀬木慎一, Kokusai/Nihon bijutsu shijō sōkan : baburu kara defure e 1990-2009 国際/日本美術市場総監：バブルからデフレ ح 1990-2009 (Panorama général du marché de l'art international et japonais de la bulle à la déflation 1990-2009), Tōkyō, Fujiwara shoten 藤原書店, 2010, p. 293-312.

La moyenne des chiffres d'affaires du « top trente » se situe à 2949 millions de yens (26,5 millions d'euros) pour l'année fiscale 2000 et 1349 millions de yens (9,7 millions) pour l'année fiscale 2005. Toutefois, il s'agit là de déclarations officielles, aussi convient-il de rester circonspect. Comme le note Raymonde Moulin :

Les prix en galerie ne sont pas transparents. [...] Une partie des transactions s'effectue dans la clandestinité et les phénomènes inquantifiables ou invisibles l'emportent sur les données apparentes et mesurables. Il existe, du fait de l'origine des œuvres et de l'argent, une économie souterraine dont l'importance est difficile à évaluer ${ }^{9}$.

Un autre constat s'impose : alors que le classement compte à chaque fois trente galeries au total, seules sept parviennent à se hisser au sommet à la fois en 2000 et 2005 , ce qui indique une forme radicale de renouvellement des effectifs $\left(\right.$ turnover $\left.^{10}\right)$. Aucune galerie n'est capable de se maintenir en tête sur la durée.

9. Raymonde Moulin, Le Marché de l'art: mondialisation et nouvelles technologies, Paris, Flammarion, 2003, p. 10.

10. Taux de turnover $=[($ Nombre de départs au cours de l'année $N+$ Nombre d'arrivées au cours de l'année N) / 2] / Effectif au $1^{\text {er }}$ janvier de l'année N. Pour obtenir un pourcentage, il suffit de multiplier cet indicateur par 100. 
En outre, même les meilleures présentent une situation précaire au regard du caractère extrêmement brusque des hausses et des baisses de bénéfices. Par exemple, en 2005, la galerie Kochūkyo 䣽中居 a bénéficié d'une hausse de $92 \%$ par rapport à 2004, mais les galeries Art Brillant, Top Art トップアート et Art Collection House アートコレクションハウス ont chuté respectivement de 44 \%, 36 \% et $35 \%$ par rapport à l'année antérieure. Ces variations très contrastées peuvent s'expliquer par le fait que le chiffre d'affaires annuel dépend fortement de la vente réussie de quelques tableaux seulement.

À côté du chiffre d'affaires, la part de l'import permet aussi de mesurer la force financière. Or, les données montrent ici clairement l'effondrement des importations suite à l'éclatement de la bulle spéculative des années 1980 . Depuis 1992, le marché japonais semble s’être replié sur lui-même, sans véritable embellie sur le long terme. Aujourd'hui comme hier, le medium « tableau » continue cependant de s'imposer comme le support principal : sculptures, multiples et objets d'antiquité ne comptent pas pour plus du huitième des ventes. Enfin, nous ne pouvons qu'être interpellés par l'importance du nombre d'œuvres en provenance de France. 
268 Cahiers d'études japonaises n²2

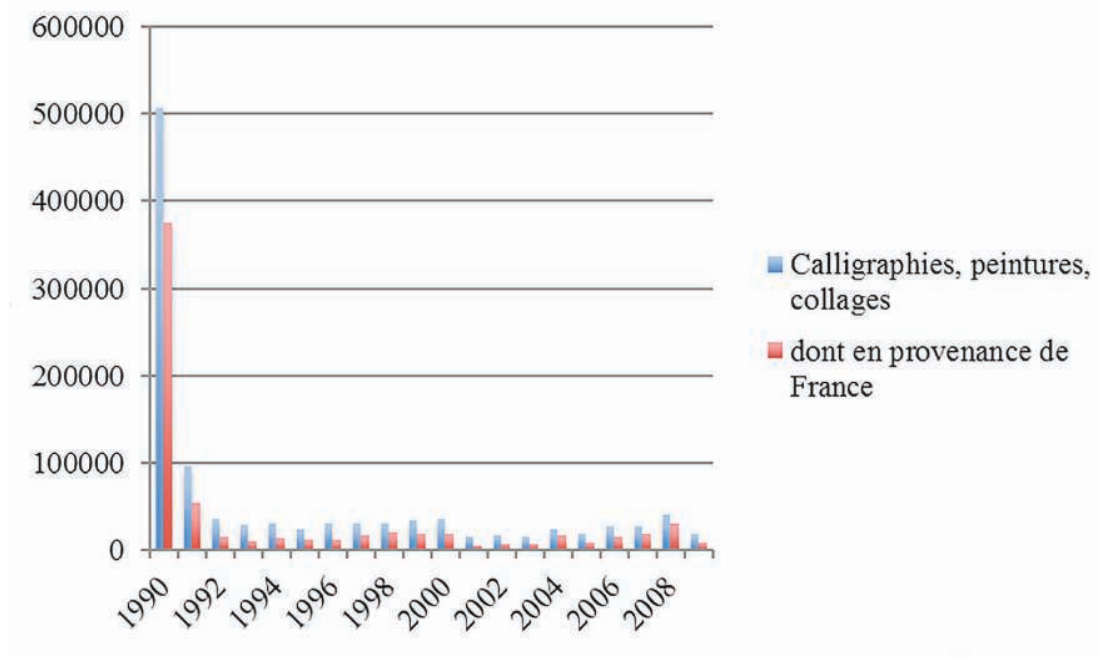

GRAPH. 1 : ÉVOLUTION DES IMPORTATIONS D'OEUVRES D’ART (1990-2009, EN MILLIONS DE YENS)

Source : Cléa Patin

TAB. 2 : IMPORTATIONS PAR TYPE D’OEUVRES POUR L'ANNÉE 2009

\begin{tabular}{|c|c|c|}
\hline & Nombre & $\begin{array}{c}\text { Montant } \\
\text { (en millions de yens) }\end{array}$ \\
\hline Gravures & 3262 & 1124 \\
\hline Sculptures & 2075 & 3448 \\
\hline $\begin{array}{c}\text { Calligraphies, peintures, } \\
\text { collages }\end{array}$ & 140131 & 18848 \\
\hline Objets d'antiquité & NR & 1632 \\
\hline $\begin{array}{c}\text { Autres (objets de curiosité, } \\
\text { timbres, etc.) }\end{array}$ & NR & 909 \\
\hline
\end{tabular}

NR : non renseigné

Sources : Segi Shin.ichi, op. cit., 2010, p. 603-605. 
Si le classement des galeries en termes de bénéfices reste instable, leur concentration sur le territoire apparaît en revanche très pérenne.

\section{Les atouts de la concentration spatiale}

Sur les 1771 galeries recensées en 2007 par l'annuaire professionnel Bijutsu nenkan, 890 se situent dans la région du Kantō et 423 dans la région du Kinki (carte 1). Ces deux zones regroupent donc à elles seules les trois quarts des marchands japonais. De plus, selon Segi Shin.ichi (entretien, 9 févr. 2007), alors que la capitale concentrait $70 \%$ des transactions dans les années 1970, elle en drainerait aujourd'hui $90 \%$. 


\section{CIPANGO}

270 Cahiers d'études japonaises n²2

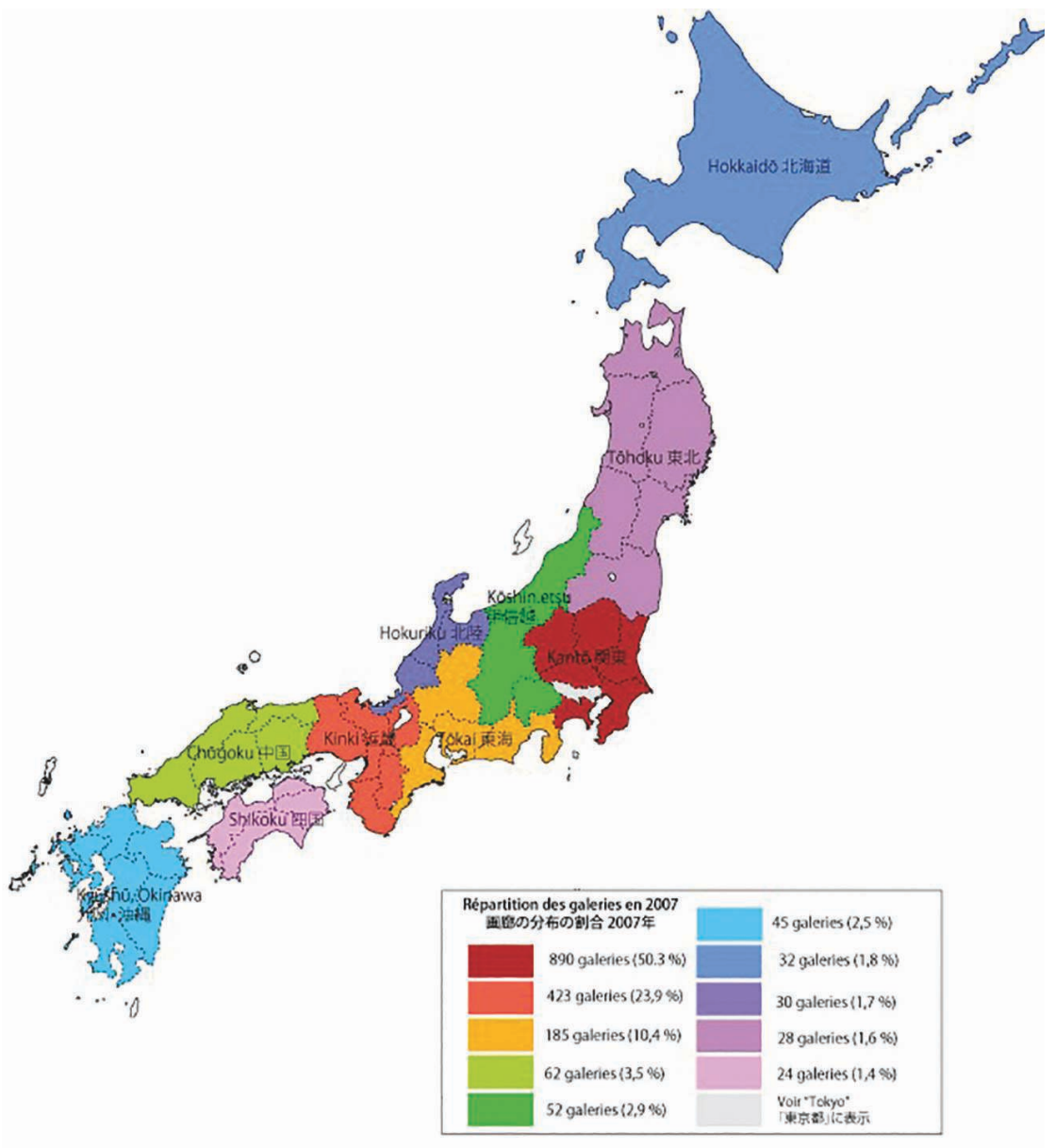

CARTE 1 : RÉPARTITION DES GALERIES AU JAPON EN 2006-2007

Source : Cléa Patin. Carte conçue à partir des données extraites de l'annuaire Bijutsu nenkan 2007. 
À Tōkyō, on trouve les galeries dans des quartiers précis, surtout entre Nihonbashi et Shinbashi avec, au centre, Ginza (cartes 2 et 3). Le fait même d'être installé à Ginza constitue un atout sur le plan concurrentiel, tant la notoriété du quartier reste forte, notamment sur le marché de l'art classé.

Dès le début, Ginza a constitué le cœur du marché de l'art. Les cinq fondateurs de notre entreprise sont originaires de ce quartier. Comme ce nom est connu de tous les clients, qu'ils habitent à Tōkyō ou qu'ils viennent des quatre coins de l'archipel, être installé ici constitue une image de marque. (Shinwa art auction シンワ・ アート・オークション) 


\section{CIPANGO}

272 Cahiers d'études japonaises n²2

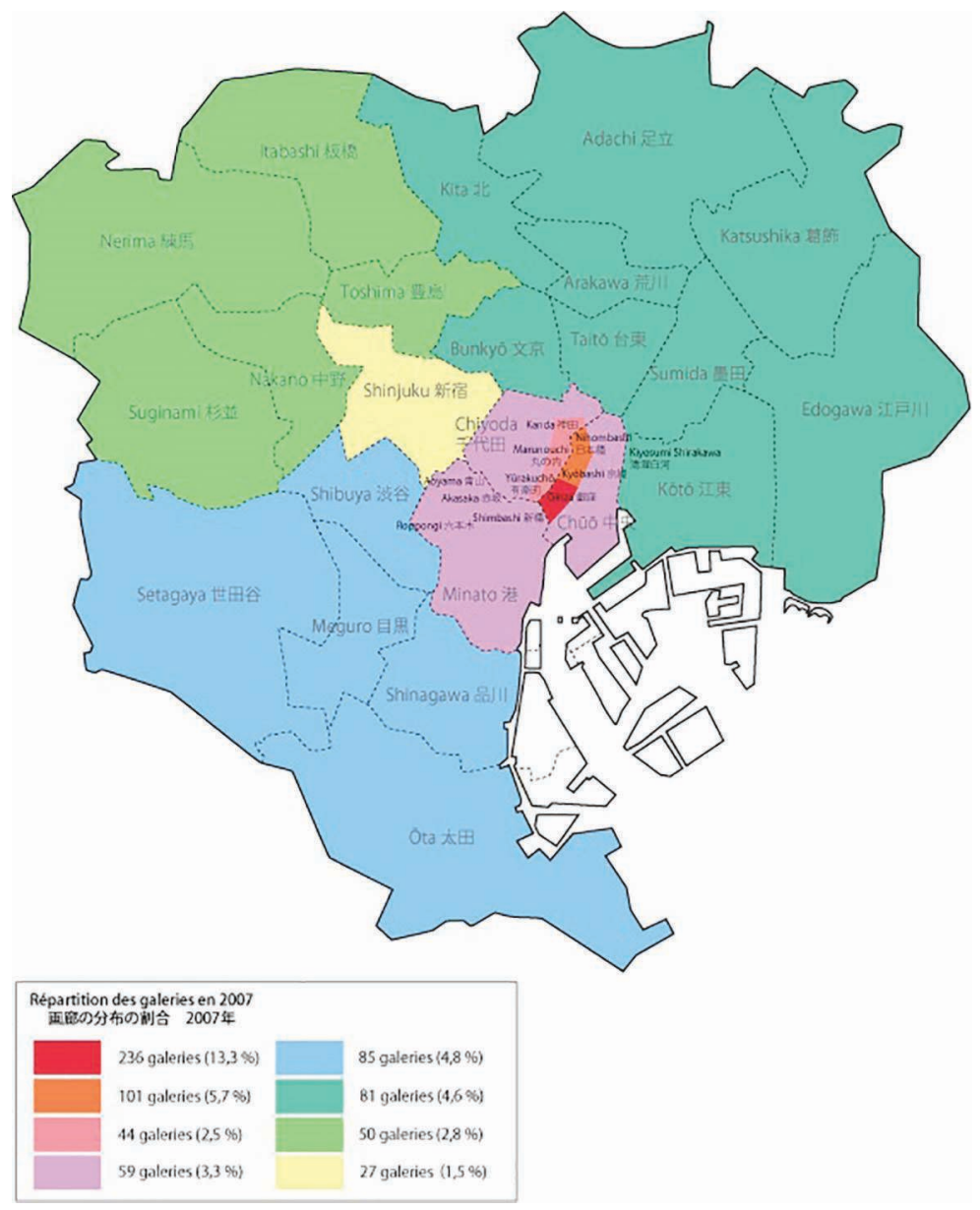

CARTE 2 : RÉPARTITION DES GALERIES SUR TŌKYŌ EN 2006-2007

Source : Cléa Patin. Carte conçue à partir des données extraites de l'annuaire Bijutsu nenkan 2007. 
Toutefois, les annuaires ne divisent pas toujours l'espace géographique de manière très pertinente. Il semble donc nécessaire de rappeler quelques évolutions récentes. Dans la deuxième moitié des années 1990, à l'image des galeries londoniennes ou new-yorkaises, un petit nombre de marchands très avant-gardistes ont reconverti des usines désaffectées et des entrepôts en salles d'exposition, à proximité du Musée métropolitain d'Art contemporain de Tōkyō, qui avait déménagé en 1994 à Kiyosumi Shirakawa. Par la suite, d'autres galeries ont été créées dans le sillage de trois grands projets de restructuration urbaine d'origine privée qui englobaient, outre la construction de logements, de bureaux et d'équipements de loisirs, la création de musées : Ebisu (1994), Roppongi (2003) et Tōkyō Midtown (2007).

Par ailleurs, cette distribution reflète-t-elle la répartition de la demande ? Si le marché de l'art s'est développé à l'origine dans les quartiers les plus peuplés, la plupart se sont transformés en zones de bureaux. Les acheteurs viennent donc plutôt des arrondissements périphériques, voire des départements voisins. À Tōkyō, si l'on analyse la densité de la population en fonction des revenus moyens, Shibuya dispose d'atouts décisifs, d'où le dynamisme de quartiers comme Ebisu ou Daikan-yama. À l'inverse, le quartier de Kiyosumi cumule à la fois de faibles densités et de faibles revenus moyens - les clients des grandes galeries commerciales n'y résident donc probablement pas. Enfin, parmi les arrondissements les plus susceptibles de fixer la demande potentielle, Setagaya et Ōta, au sud, présentent des populations stables financièrement, avec un fort taux d'emploi à durée indéterminée et de dirigeants d'entreprises. 


\section{CIPANGO}

274 Cahiers d'études japonaises n²2

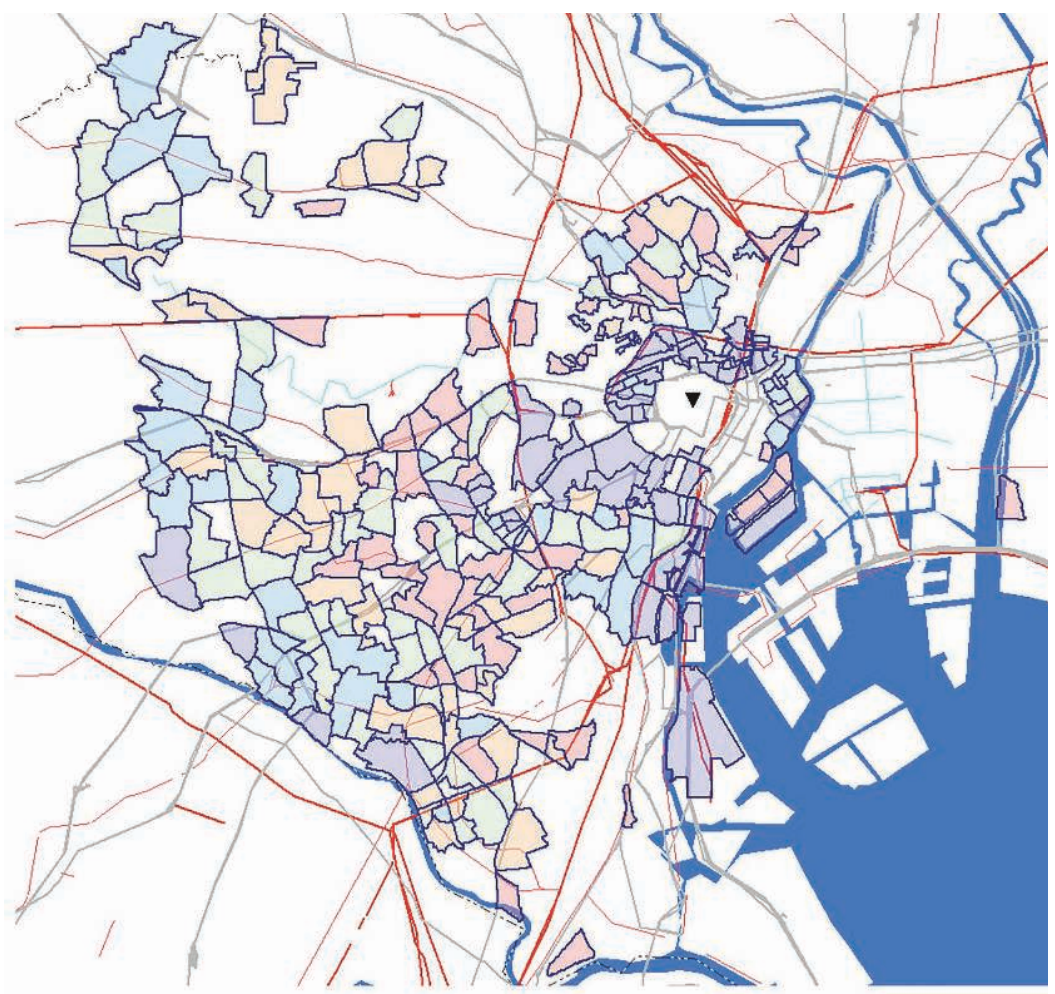

Densité $>19000$ habitants $/ \mathrm{km} 2$

$16000<$ Densité $<19000$

$13000<$ Densité $<16000$

$10000<$ Densité $<13000$

Densité $<10000$ habitants $/ \mathrm{km} 2$

CARTE 3 : RÉPARTITION DE LA TRANCHE DES MÉNAGES LES PLUS RICHES (30\%) À TŌKYŌ EN 2005 (REVENUS MOYENS > 6,5 MILLIONS DE YENS PAR AN) Source : Cléa Patin. Données du recensement de 2005 mises en forme par l'agence immobilière Seesa Inc. 
La concentration du marché de l'art ainsi que le regroupement des galeries par quartier peut s'expliquer par la présence de bénéfices retirés des « économies d'agglomération $\gg^{11}$, terme qui désigne à la fois les avantages liés au fait même d'être situé dans une grande ville (économies d'urbanisation), et les gains retirés d'une concentration d'entreprises agissant dans une même sphère d'activité (économies de localisation).

Les économies de localisation notamment exercent une influence décisive sur la formation des réseaux informationnels à l'origine du jugement sur la valeur des biens d'art. En effet, aucune œuvre n'a de valeur dans l'absolu ${ }^{12}$ : seule la comparaison relative permet aux acquéreurs potentiels de s'informer sur sa nature, son prix et sa qualité. Dans le vocabulaire des galeristes, il faut « former son œil », fixer ses préférences en accumulant une masse considérable de données. Ces flux informationnels sont d'autant plus importants que le système entier repose sur une action et une production très variables (flexibilité et caractère intermittent des emplois artistiques), ainsi que sur une innovation incessante (situation spécifique de «surproduction » liée à l'incertitude du succès et à la nécessité du renouvellement très rapide des produits). Les galeries partagent un même monde, font partie d'un même « biotope ». Leurs employés se surveillent et se rendent mutuellement visite. Ils collectent l'information et la font circuler, à travers des mécanismes de type «bouche à oreille », tout en cherchant à en identifier la valeur, à en tester le degré d'actualité et de résonance, à travers une relation interpersonnelle. Ils sont aussi leurs propres fournisseurs et clients. Le regroupement dans un même quartier, voire dans un même immeuble, permet de partager des frais de promotion et favorise l'organisation d'événements en commun (Aozora de āto 青空でアート, Ginza night 銀座ナイト, etc.).

11. Brendan O’Flaherty, City Economics, Massachusetts, Harvard University Press, 2005, pp. 16-25.

12. Voir Pierre-Michel Menger, Le Travail créateur : s'accomplir dans l'incertain, Paris, Gallimard-Seuil, Éditions de l'EHESS, 2009. Ainsi que Pierre-Michel Menger, « Les artistes en quantités. Ce que sociologues et économistes s'apprennent sur le travail et les professions artistiques », Revue d'économie politique, vol. 120, janvier 2010, Dalloz, p. 205-236. 
276 Cahiers d'études japonaises n²2

Après l'évolution des galeries dans l'espace, voyons comment elles se comportent dans le temps, au niveau des variations de population et du cycle de vie.

\section{Évolution dans le temps : déclin démographique et sensibilité à la conjoncture}

La majorité des galeries sont nées au cours des années 1980, notamment dans la région du Kantō. De manière générale, on assiste à une baisse du nombre de fondations depuis 1990.

Le nombre total de galeries suit une courbe ascendante très forte entre 1987 et 1992, passant de 1786 à 2045 établissements (graph. 2). Une année après l'éclatement de la bulle spéculative, début 1992, on compte encore 942 galeries en programmation pure, 124 galeries en semi-programmation et 444 galeries locatrices. À l'époque, la plupart des acteurs pensaient en effet qu'il s'agirait d'une crise passagère et ont tenté de poursuivre leur activité, d'où un décalage de deux ans environ entre l'éclatement de la bulle et ses contrecoups sur le marché de l'art. Par rapport à ce sommet, l'année 2007 présente des baisses de $27 \%, 5 \%$ et $33 \%$ respectivement pour les galeries programmatrices, semiprogrammatrices et locatrices. En pleine période de récession et de restructuration - « la décennie perdue »- de nombreuses galeries disparaissent. La pente de la courbe suggère même que le choc de la récession ait touché davantage les galeries programmatrices, qui ne bénéficient pas d'un revenu foncier. De plus, alors que l'on aurait pu s'attendre à une embellie à partir de 2003, suite à l'amélioration de l'environnement macro-économique, le déclin démographique des galeries se poursuit jusqu'en 2007. 


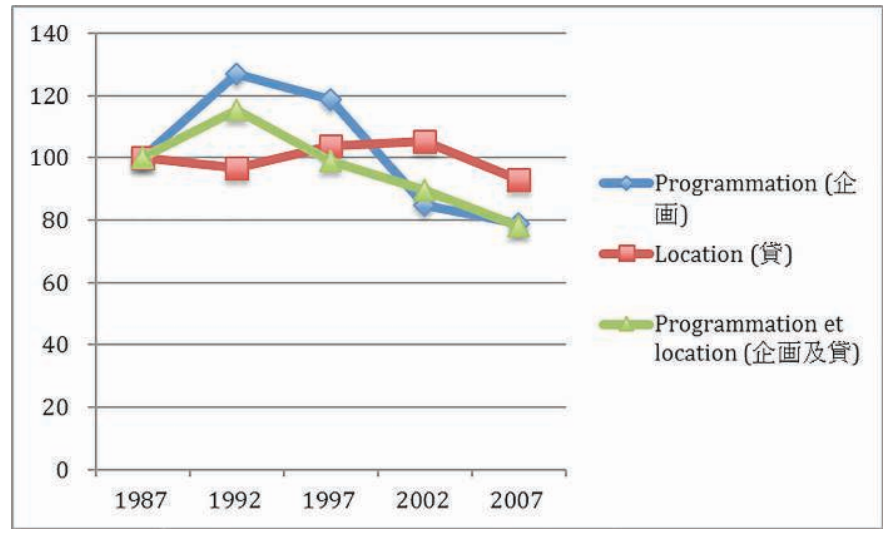

GRAPH. 2 : ÉVOLUTION DÉMOGRAPHIQUE DES GALERIES PAR TYPE SUR L'ENSEMBLE DU TERRITOIRE (INDICE BASE 100 : 1987) Source : Cléa Patin. Données extraites de l'annuaire Bijutsu nenkan

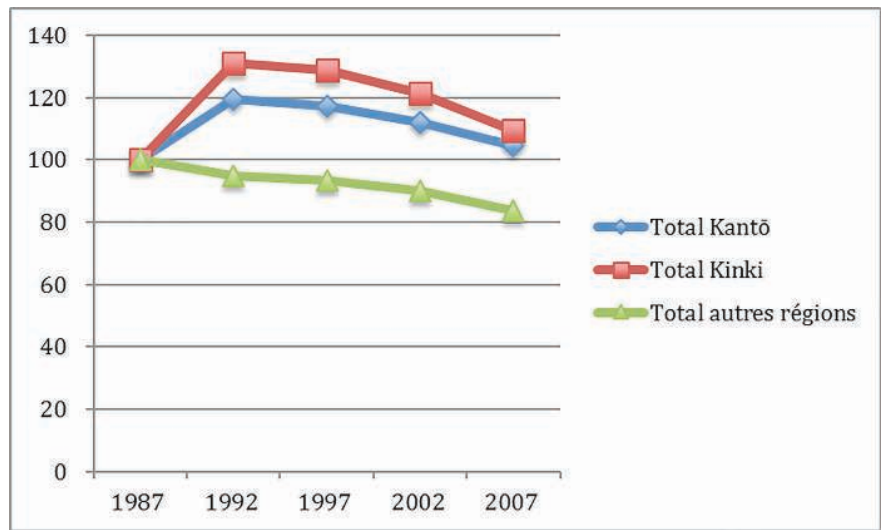

GRAPH. 3 : ÉVOLUTION DÉMOGRAPHIQUE DES GALERIES PAR RÉGION (INDICE BASE $100:$ 1987)

Source : Cléa Patin. 
Toutefois, il faut prendre en compte le biais du «pari réussi » : le taux de disparition pourrait s'avérer beaucoup plus élevé, mais compensé par un nombre important de nouveaux entrants, ce qui masquerait l'existence d'un cycle de vie extrêmement rapide. Pour avoir une vision plus objective du taux de mortalité, il faut donc quantifier les apparitions et les disparitions, en comparant le nom des galeries selon les cinq dates considérées. C'est ce que nous avons fait pour Ginza et Kyōbashi.

TAB. 3 : CYCLE DE VIE DES GALERIES ENTRE 1987 ET 2007

À GINZA ET KYŌBASHI

\begin{tabular}{|c|c|c|c|c|c|c|}
\hline & & 1987 & 1992 & 1997 & 2002 & 2007 \\
\hline Ginza & Indice de turnover & $/$ & $24 \%$ & $22 \%$ & $19 \%$ & $/$ \\
\hline & $\begin{array}{c}\text { Chgt du nom de la } \\
\text { galerie }\end{array}$ & 3 & 3 & 3 & 0 & 0 \\
\hline & Chgt de direction & 0 & $5(5)$ & $6(5)$ & $6(2)$ & $5(1)$ \\
\hline Kyöbashi & Indice de turnover & $/$ & $29 \%$ & $35 \%$ & $26 \%$ & $/$ \\
\hline & $\begin{array}{c}\text { Chgt du nom de la } \\
\text { galerie }\end{array}$ & 2 & 1 & 1 & 0 & 0 \\
\hline & Chgt de direction & 0 & $1(1)$ & 1 & 0 & 0 \\
\hline
\end{tabular}

Note : entre parenthèses sont indiqués les cas où, lors d'une succession, la relève est prise par un membre de la même famille.

Source : Données extraites de l'annuaire Bijutsu nenkan.

Le nombre de disparitions fait plus que doubler entre 1992 et 1997 (il passe de 43 à 102), avant de se stabiliser légèrement (hausse de $10 \%$ jusqu'en 2002), puis reculer (baisse de $40 \%$ avant la crise des subprimes de septembre 2008). En revanche, ces fermetures se trouvent compensées par un nombre élevé d'apparitions : 94 galeries en 1992, 82 en 1997, 82 en 2002 et 49 en 2007. Par ailleurs, 171 ont fait faillite à moins de cinq ans d'intervalle. 57 galeries n'ont tenu que cinq ans. Le cycle de vie apparaît donc très court, avec un turnover situé en moyenne à $21 \%$ pour Ginza et $30 \%$ pour Kyōbashi entre 1992 et 2002.

À cela s'ajoutent dix déménagements repérés de Ginza vers Kyōbashi. De fait, les migrations sont fréquentes. À Tōkyō, après la bulle spéculative, étroitement 
corrélée à la hausse des prix du foncier et de l'immobilier ${ }^{13}$, de nombreux établissements ont quitté les cinq arrondissements centraux. En outre, au cours des années 1990, alors que l'avenue centrale de Ginza (Chūō dōri 中央通り) se transformait peu à peu en vitrine des grandes marques européennes, beaucoup ont aussi déserté les emplacements en rez-de-chaussée pour s'éparpiller dans les rues adjacentes, en sous-sol ou dans les étages supérieurs. Les quartiers avoisinants de Kyōbashi et Nihonbashi, quoique encore situés dans l'arrondissement central de Chūō, ont alors offert des loyers plus avantageux.

Ma galerie était à Ginza, mais le bâtiment a été démoli pour être reconstruit à neuf et on m'a demandé de m'en aller. J'ai d'abord pensé rester dans le même quartier. Malheureusement, le loyer était trop élevé. Alors j'ai migré ici, à Kyōbashi. Sans doute était-ce un mal pour un bien : avant, ma galerie était deux fois plus petite. Et puis, quand j'ai été mis à la porte, j'ai reçu une indemnité d'environ 1 million de yens. Lors des négociations, j'ai un peu surestimé les coûts, tout en limitant au maximum les dépenses. J’ai donc évité un très gros déficit. (Galerie T-Boxティー・ボックス)

Si les données macroéconomiques sur les galeries au Japon mettent en lumière un milieu soudé mais fragile, fortement concentré autour de deux grands pôles (le Kantô et le Kansai), elles sont à mettre en regard avec le dynamisme et les limites rencontrés sur le terrain.

\section{Une étude de cas : le fonctionnement au quotidien des galeries tōkyōites}

Afin de saisir plus en détail les modes de gestion des galeries japonaises, nous avons procédé à un travail d'enquête. Dans un premier temps, nous avons sélectionné les galeries de l'échantillon, en confrontant les listes fournies par trois annuaires: Bijutsu nenkan, Bijutsu techō nenkan 美術手帳年鑑 et Gekkan Gallery 月刊ギヤ

13. Shimizu Chihiro, "Pricing Structure in Tokyo Metropolitan Land Markets and its Structural Changes: Pre-Bubble, Bubble, and Post-Bubble Periods", Journal of Real Estate Finance and Economics, nov. 2007, vol. 35, n 4, pp. 475-496. 
280 Cahiers d'études japonaises n²2

ラリー (toutes les galeries retenues se trouvent ainsi répertoriées dans au moins deux d'entre eux). L'utilisation conjointe de ces guides, dont les bases ne sont pas toujours homogènes (Gekkan Gallery tend à privilégier des galeries plus engagées dans l'art international), a permis d'assurer une meilleure représentativité. Si la très forte concentration du marché de l'art plaidait en faveur d'un recentrage sur Tōkyō, il s'agissait aussi d'élaborer un échantillon susceptible de refléter la proportion de programmation et de location, le niveau d'engagement dans l'art international, le degré de visibilité et d'ancienneté ainsi que la répartition géographique des galeries Tōkyöites. Avec l'aide de deux représentants de la profession et un collectionneur, nous avons finalement retenu 291 galeries. Le taux de réponse final se situe à $37,5 \%$ (106 réponses), ce qui paraît satisfaisant, compte tenu du caractère approfondi des questions et du renvoi à la charge des enquêtés.

Notre questionnaire comprend au total 32 questions, qui permettent d'évaluer le profil juridique et les coûts fixes des galeries, leurs relations aux artistes et aux collectionneurs, avant d'acquérir une vision d'ensemble de leur structuration en tant que groupe professionnel. Dans l'ensemble, les résultats de l'enquête reflètent les enseignements sur la place globale des galeries : il s'agit de micro-entreprises fragiles, mais au centre des réseaux de formation de la valeur.

\section{Des «micro-entreprises »à l'existence éphémère}

\section{$\underline{\text { Statuts juridiques et gestion des coûts fixes }}$}

Pour ce qui est de la structure juridique, la situation des enquêtés ne reflète pas encore le changement de la loi sur le statut des entreprises du $1^{\text {er }}$ mai 2006. $41 \%$ des établissements affichent donc le statut de société anonyme, signe de stabilité, $31 \%$ sont des sociétés à responsabilité limitée, tandis que $28 \%$ renvoient à des structures plus avantageuses sur le plan fiscal, mais plus fragiles, comme des associations, des sociétés civiles à personnalité juridique, des sociétés en commandite ou en nom collectif. Le statut juridique semble en outre positivement corrélé à la date de fondation : les sociétés anonymes concernent davantage les galeries créées dans les années 1960 et 1970, tandis que les sociétés à responsabilité 
limitée progressent dans l'échantillon à partir des années $1980^{14}$. L'opposition entre sociétés anonymes et autres structures mérite cependant d'être tempérée : les galeristes minimisent, au cours des entretiens, l'impact des choix organisationnels pour percer le marché.

Du côté des coûts fixes, seulement $13 \%$ des galeries présentent des filiales ( $11 \%$ au Japon et $2 \%$ à l'étranger). Quant à la surface, elle s'établit à moins de $100 \mathrm{~m}^{2}$. Sans doute atteint-on là un plafond pour la ville de Tōkyō. Enfin, dans $80 \%$ des cas, les galeristes ne sont pas propriétaires de leur lieu de travail. Détenir le foncier semble être l'apanage des galeries à forte proportion de location.

\section{Un équilibre financier précaire}

Le taux de non-réponse à la question du chiffre d'affaires concerne presque le quart des enquêtés (23\%), soit par répugnance à avouer des difficultés financières (au cours des entretiens, la majorité des galeries avouent surnager, à la « limite du déficit »), soit par une crainte d'éveiller l'attention du fisc. Une remarque cependant : au sommet ne subsistent que des galeries en programmation pure. Si la location apporte une certaine stabilité, elle ne concède pas de revenus importants sur le long terme : la moitié des galeries partiellement locatrices n'engrangent pas plus de 20 millions de yens (140 000 euros).

Paradoxalement, le sujet de l'endettement s'est avéré moins problématique (le taux de non-réponse a plafonné à $10 \%)$. Or, il nous renseigne sur la fragilité potentielle en cas de retournement de la conjoncture, de même que sur la force

14. Les sociétés à responsabilité limitée yügen gaisha 有限会社 (YG), créées par une loi de 1940, étaient jusqu'en 2006 majoritairement utilisées par les PME - d'où l'importance de ce statut à un moment où, de manière générale, le nombre de galeries s'accroît sur le marché. Cependant, avec la réforme récente des sociétés anonymes kabushiki gaisha 株 式会社 (KG), via la nouvelle loi sur les sociétés (Kaisha hō 会社法), entrée en vigueur le $1^{\text {er }}$ mai 2006, le statut de YG a été supprimé et le régime des KG considérablement simplifié : désormais le capital minimum requis pour la création d'une KG est de 1 yen, contre 10 millions auparavant, tandis que le nombre minimum d'administrateurs est passé de 3 à 1 . Aujourd' hui, toute nouvelle galerie a donc plutôt intérêt à se lancer directement en tant que KG. 
282 Cahiers d'études japonaises n²2

financière, mesurée à l'aune de la crédibilité auprès des organismes financiers. Dans l'échantillon, le taux d'endettement passe d'ailleurs de $30 \%$ pour les galeries à faibles revenus, à $66 \%$ pour celles revendiquant un bénéfice supérieur à 100 millions de yens (700 000 euros) par an.

Tous types confondus, la majorité des galeries peine cependant à équilibrer la balance entre les revenus et les dépenses et avouent surnager, toujours à la « limite du déficit ». À l'image des artistes qu'elles soutiennent, beaucoup dépendent des versements venant d'amis ou de proches.

Pour ce qui est de la rentabilité... Ça fait plus de dix ans que je suis en quasi déficit (c'en est presque risible). Presque pas de bénéfices... Je suis obligée de vendre de temps en temps des pièces de ma collection personnelle pour renflouer les comptes. (Galerie Soh)

Chez nous, ni bénéfice, ni déficit. Nous parvenons tout juste à nous maintenir. Notre chiffre d'affaires avoisine les cent millions de yens, mais si l'on retire les frais relatifs au loyer ainsi que les charges (publicité, présentations, fournitures pour les artistes), il ne reste presque rien. Actuellement, nous sommes en programmation pure. Toutefois, il se pourrait bien que nous revenions en location partielle. (Galerie Marunouchi)

Vous me demandez si je m'en sors ? La réponse est non. Le déficit de la galerie est renfloué par les revenus de mon mari, professeur à l'université. Moi aussi, venant d'une famille aisée, je bénéficie d'un peu de réserves personnelles. Pour continuer à fonctionner, il faut puiser dans son épargne. Mais mon but n'est pas de faire du chiffre. Ma recherche du Beau est anti-économique. (Galerie Ippodō 一穂 堂)

Le travail de galeriste ne repose fondamentalement pas sur l'idée de gagner de l'argent. Un marchand doit toujours passer en second, après ses clients et ses artistes. (Galerie Kotōken) 


\section{L'omniprésence de la direction}

Toutes les galeries présentent des effectifs minimalistes. Dans $41 \%$ des cas, les directeurs gèrent seuls l'entreprise, cumulant toutes les charges et fonctions. Quand ils sont secondés, c'est surtout par des employés à temps partiel ou en arubaito (moins de cinq en moyenne). La compétitivité même de la galerie dépend donc de la qualité de leur « œil avisé », de leur flair, qui leur fait découvrir avant d'autres les valeurs artistiques de demain.

C'est bien parce que j'agis en faisant confiance à ce que je vois, sans écouter les conseils des autres, que ça fonctionne. Un directeur est responsable de ses choix, qu'ils conduisent à des succès ou des déceptions. D'ailleurs, personne ne peut savoir à l'avance qui va réussir. (Galerie Shirota)

Je ne choisis pas en me fondant sur des livres, je fais confiance à mes yeux, à mes mains, à mes cinq sens. (Galerie Ippodō)

D'où l'intérêt de connaître leurs motivations.

TAB. 4 : LES RAISONS DE LA FONDATION (NOMENCLATURE PAR THÈMES)

\begin{tabular}{|c|c|c|}
\hline A. Succession & $\%$ & $\begin{array}{c}\text { Nombre de } \\
\text { réponses }\end{array}$ \\
\hline A1 Héritage familial & $10 \%$ & 11 \\
\hline A2 Succession du précédent directeur & $10 \%$ & 11 \\
\hline $\begin{array}{c}\text { A3 Prise d'indépendance après avoir travaillé dans } \\
\text { une autre galerie }\end{array}$ & $8 \%$ & 9 \\
\hline $\begin{array}{c}\text { A4 Lieu d'exposition lié à la reconstruction/ } \\
\text { rénovation de l'immeuble }\end{array}$ & $5 \%$ & 5 \\
\hline B. Autonomie, plaisir & $14 \%$ & 15 \\
\hline B1 Amour de l'art & $3 \%$ & 3 \\
\hline B2 Expérience de collectionneur & $8 \%$ & 9 \\
\hline B3 Sentiment d'autonomie/d'initiative & & \\
\hline
\end{tabular}




\begin{tabular}{|c|c|c|}
\hline B4 Épanouissement personnel & $15 \%$ & 16 \\
\hline \multicolumn{3}{|l|}{ C. Diffusion de l'art } \\
\hline C1 Diffusion de l'art à l'ensemble de la société & $14 \%$ & 15 \\
\hline C2 Diffusion de l'art japonais à l'étranger & $5 \%$ & 5 \\
\hline \multicolumn{3}{|l|}{ D. Soutien aux artistes } \\
\hline $\begin{array}{c}\text { D1 Promotion de l'art contemporain, des jeunes } \\
\text { artistes }\end{array}$ & $25 \%$ & 26 \\
\hline D2 Soutien et accompagnement des artistes & $19 \%$ & 20 \\
\hline Non réponse & $27 \%$ & 29 \\
\hline Total & $100 \%$ & 106 \\
\hline
\end{tabular}

La majorité des enquêtés (44\%) revendiquent d'abord un soutien aux artistes. L'idée selon laquelle le galeriste est là pour soutenir, aider, former, semble profondément enracinée. Le terme même d'éduquer ou de fortifier (kitaeru 鍛え る) revient très souvent dans les discours, au risque parfois d'agacer les plasticiens, qui en dénoncent l'aspect infantilisant et paternaliste ${ }^{15}$.

Ensuite, sur un mode un peu différent puisqu'il s'agit de la diffusion de l'art, donc du soutien à l'art en soi plus qu'à des individus en particulier, vient la volonté altruiste, un peu naïve sans doute, de « propager l'art à l'ensemble de la société ». Cela reflète probablement l'image, valorisée au Japon, du dévouement à la collectivité. Toujours est-il que $14 \%$ des enquêtés affirment agir pour le bien communautaire, de manière désintéressée.

Face à la notion de service aux autres, des motivations plus individuelles, comme l'épanouissement personnel et le désir d'indépendance, tiennent aussi une place clé : le métier de galeriste assouvit une curiosité intellectuelle toujours en éveil, par l'autoformation permanente, et procure un prestige social particulièrement élevé, du fait de la position dominante qu'occupe la culture dans l'imaginaire collectif.

15. Nous renvoyons à la conférence de Hatakeyama Kōji 畠山耕治 (artiste), intitulée 《Bijutsu sakka no kakeibo » 美術作家の家計簿 (Carnet de dépenses d'un artiste), organisée le 23 mars 2007 par AIT (Art Interactive Tōkyōアート・インタラクティヴ 東京) dans le cadre du cycle «Bijutsu korekutā no gimon ni kotaete » 美術コレクタ 一の疑問に答えて (Réponses aux questions des collectionneurs). 
Enfin viennent des considérations successorales ou patrimoniales $(21 \%$ des galeristes). Ainsi, $10 \%$ évoquent un proche déjà actif sur le marché de l'art, tandis que $8 \%$ affirment avoir pris leur indépendance après une longue période de formation et de travail auprès d'autres confrères. Ces relations croisées, soit dans l'attente de retrouver leur galerie d'origine (dans le cas des transmissions familiales), soit dans le but de créer une nouvelle galerie, renforcent les liens de dépendance et de loyauté entre galeristes et favorisent la diffusion de l'information. Enfin, une toute petite minorité, propriétaire des locaux, a indiqué que la rénovation de l'immeuble avait déclenché leur décision de devenir galeriste. Il s'agit alors de rentabiliser les frais de travaux en faisant porter une partie des coûts sur les artistes, à travers la perception d'un loyer.

Quand j'ai voulu ouvrir ma galerie à Ginza, au début des années 1980, on m'a fait remarquer qu'il était impossible pour une débutante comme moi de percer le monde de l'art traditionnel. Ce milieu a ses traditions : il faut d'abord entrer dans une galerie comme employé - comme « premier commis » - et y faire ses preuves pendant cinq, dix, quinze, vingt ans... Alors seulement on peut songer à prendre son indépendance. C'est la manière japonaise. Toutefois, du côté de l'art contemporain, comme le genre était totalement nouveau, ce fonctionnement n'avait pas encore court. (Galerie Soh)

Le fait que la galerie dépende très fortement des choix effectués à son sommet par un seul(e) homme (femme) constitue certes un atout, par la cohérence des sélections que cela suppose, mais pose aussi un problème : il s'agit d'une hérésie en terme de diversification des risques. Or, le risque est omniprésent sur tout le segment de la production. 


\section{La relation aux ouvres et aux artistes : des négociations effectuées dans l'incertitude}

\section{Tendances artistiques: des choix conservateurs?}

Pratiquement tous les galeristes ( $85 \%$ ) déclarent « faire de l'art contemporain » - une manière de profiter des effets de distinction sur les thèmes à la mode -, mais dans les faits, des choix plus conservateurs sont à l'œuvre, sans doute pour limiter les risques encourus. On observe en effet une variété de choix esthétiques qui, parallèlement à la force financière, conditionnent les modes de promotion des artistes.

Ainsi, $16 \%$ indiquent vendre de la peinture moderne occidentale, $58 \%$ de la peinture nihonga et $50 \%$ de la peinture yōga. En outre, le quart de l'échantillon apparaît actif à la fois sur le premier et le second marché. Dans ce cas, les œuvres acquises sur le marché secondaire n'apparaissent pas forcément en devanture : elles circulent le plus souvent dans l'arrière-boutique ou au sein des «sociétés d'échanges » (kōkankai).

Parmi les sous-catégories de l'art contemporain, le support le plus prisé reste la photographie ( $58 \%$ des galeries). Les installations bénéficient d'un soutien solide (36\%), mais les performances, faute sans doute d'un espace suffisant, restent à la traîne (17\%). Dans l'ensemble, l'art vidéo atteint un score honorable (31 \%), quand bien même il s'agit de l'un des segments les plus déficitaires du marché. On note également une diffusion très large pour la gravure (69\%) et la sculpture (66\%), deux domaines qui peuvent relever aussi bien de l'offre contemporaine que de l'art ancien (ukiyoe, sculpture figurative). L'artisanat d'art (33\%) demeure un élément important du marché. Quant à la calligraphie, elle apparaît en reste (16\%), du fait de son statut un peu hybride ${ }^{16}$ et de l'existence de réseaux de diffusion parallèles, tels que les expositions financées par les élèves des écoles reconnues.

16. Laïli Dor, « Revaloriser la calligraphie : le rôle des expositions pendant les ères Meiji et Taishō », Ebisu, n 50, automne-hiver 2013, p. 135-159. 


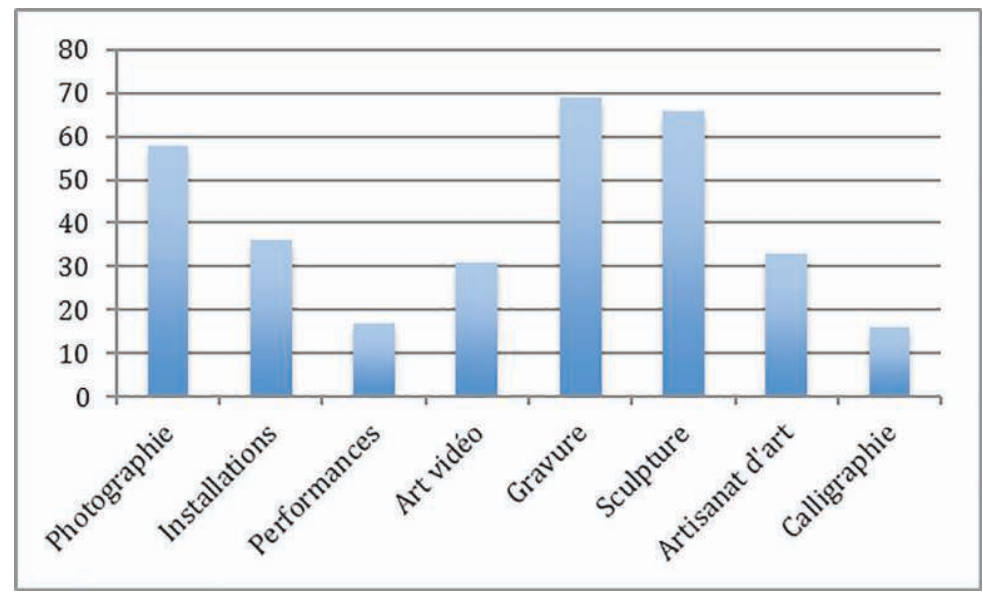

GRAPH. 4 : SOUS-CATÉGORIES DE L'ART CONTEMPORAIN (EN \%)

Source : Cléa Patin.

Le nombre d'expositions, toutes tendances artistiques confondues, s'établit autour de seize par an, en moyenne et par galerie, soit pratiquement une exposition toutes les trois semaines. En leur sein, plus que tout autre élément, le prix revêt une dimension stratégique et symbolique.

\section{Enjeux autour de la fixation des prix}

Dans l'ensemble, les prix des œuvres en vente (tableaux, photographies, gravures, etc.) se répartissent dans les catégories moyennes : entre 40000 et 200000 yens ( $49 \%$ des galeries) ou entre 200000 et 1 million de yens ( $40,5 \%$ ). Force est de constater qu'il s'agit d'une fourchette modeste en comparaison d'autres marchés nationaux (chinois et américain notamment). Les galeries programmatrices négocient la fixation des prix avec les artistes au cas par cas. Or, déjà complexe dans le cas des tableaux, cette question se complique encore dans le cas des multiples (gravures, photographie et art multimédia). Malgré la numérotation des œuvres, celles-ci résistent en effet au contrôle stratégique de la rareté. 
Actuellement, je tire mes photographies à huit exemplaires en moyenne, avec des prix autour de 500 000, 600000 yens. Ça me paraît une somme importante. Mais au fond, je ne sais pas vraiment si c'est cher ou pas. Je ne comprends pas grand-chose aux prix des photographies. (Artiste photographe I)

La fixation des prix obéit cependant à une règle d'or : il vaut toujours mieux fixer un prix de départ modeste, pour permettre une augmentation progressive, mais soutenue. En effet, une inflexion à la baisse, même minime, constitue un signe très négatif sur le marché ${ }^{17}$. Ainsi, si, dans de rares cas, des marchands bien établis peuvent recourir à des procédés artificiels pour faire monter la cote de leurs protégés, en portant par exemple leurs œuvres aux enchères, ce comportement s'avère à double tranchant : un échec en vente publique risque de disqualifier l'artiste à jamais.

Pour le prix, c'est moi qui décide. Je dis toujours aux jeunes artistes de démarrer avec des prix bas. En effet, l'art constitue une exception : on ne peut pas baisser les prix quand ils sont trop élevés. Par contre, tout le monde est content quand ils augmentent progressivement, à commencer par les acheteurs. Alors je négocie ferme avec les artistes, qui suivent mes conseils de bonne grâce. (Galerie Tōkyō 東京画廊)

\section{Quand l'absence de contrat constitue la règle}

Face à de possibles abus de la part des galeristes comme des artistes, le nombre de contrats écrits apparaît extrêmement faible. Ils concernent principalement des galeries locatrices, dont on peut penser qu'elles se soucient davantage des modalités de bail que du contrôle - et a fortiori du monopole - sur la production artistique. Seulement $2 \%$ des galeries de l'échantillon indiquent négocier des « contrats d'exclusivité », tandis que $60 \%$ se contentent d' « accords verbaux »,

17. Raymond Moulin, op. cit., 1992, p. 49. 
voire « tacites ». Certes, la décision de clarifier à l'écrit les conditions de vente dépend d'un rapport de force : la plupart des galeristes ne prennent pas la peine d'établir des contrats rigoureux tant que leurs protégés ne sont pas célèbres. Toutefois, si l'exclusivité peut être négociée à l'oral, la prise en dépôt devrait obliger à plus de précaution. Des litiges peuvent en effet survenir en cas de disparition, de vol ou de détérioration des œuvres. De même, les expositions à l'étranger, sources d'investissements importants, posent la question de la conservation et du partage des coûts (location d'une salle dans un hôtel, transport, billetterie, etc.). Cependant, même dans ces cas extrêmes, les relations de confiance prédominent.

J'évite absolument les contrats avec les artistes. Je ne fonctionne qu'aux relations de confiance. (Galerie Kotōken)

Autrefois, lors des commandes avec des versements anticipés, on établissait des contrats. Mais aujourd'hui, c'est impensable. Moi, je gère les choses de manière tacite. C'est la règle : la galerie protège son peintre, le peintre soutient sa galerie. (Galerie Kamakura drowing)

Je n'établis que des accords oraux. Mais une promesse est une promesse. En programmation, les artistes doivent me prévenir s'ils veulent agir avec un autre marchand. S'ils le font sans m'en parler, alors je leur coupe mon soutien. (Galerie T-Box)

Dans notre monde, pas de contrat. Pourquoi ? Parce que la majorité des transactions s'effectue en espèces ! Pas besoin de contrat. Au contraire, ça ruinerait les relations. Avec les artistes, on est liés mutuellement... Mieux vaut négocier de manière informelle. Laissons les contrats à une poignée d'Américains. (Galerie Tōkyō)

Nous commençons toujours par un accord verbal, avant de formaliser éventuellement les choses. Mais de toute façon, on ne peut pas savoir à l'avance qui va réussir. Pas la peine d'établir un contrat si on n'arrive pas à vendre. Par contre, si l'un de nos artistes expose ailleurs, c'est par notre intermédiaire. (Galerie Marunouchi) 
Personne ne sait si j'ai un contrat écrit avec mes artistes, mais je les suis depuis tant d'années... Aucun professionnel n'oserait les contacter directement. (Galerie Soh)

L'absence de contrat révèle une fois de plus la profondeur des relations de confiance, de solidarité, ou encore un mécanisme de pouvoir, qui se nouent dans le milieu. Mais il s'agit aussi d'un élément de fragilisation non négligeable en cas de contentieux. Or, nul établissement n'est à l'abri de dérapages et de conflits, même si ces tensions sont parfois niées, pour ne pas perdre la face :

Quand des problèmes surgissent dans le monde de la peinture nihonga, les relations se distendent naturellement. Un galeriste ne peut critiquer ouvertement un peintre. De son côté, l'artiste ne refuse jamais clairement de peindre, même s'il fait la grève des pinceaux. On continue de se saluer, comme si de rien était, en souriant. Quel monde redoutable! (Marchand de gravures)

Première cause de conflits, l'immixtion d'un tiers dans la relation duelle galerie-artiste. Voici le témoignage d'un marchand du côté des « pirates », dont la légèreté à l'égard des galeries leaders a embarrassé plus d'un.

Je travaillais avec les mêmes artistes que les grandes galeries, parce qu'elles n'avaient pas d'exclusivité pure et dure. J'arrivais à faire des petits trucs à part. Donc c'était un peu du piratage. Mais je m'entendais bien avec les artistes. Par exemple, Kusama ${ }^{18}$ m'a dit de vendre ses sculptures à Robert Miller, sa galerie newyorkaise. Ils m'ont dit : « Mettez-nous l'édition complète en dépôt et on va la vendre ; on vous les paiera au fur et à mesure ». Financer 100 bronzes de ça ? J'ai le temps de faire 25 fois faillite ! J'ai expliqué ça à Kusama. Elle m'a donc dit d'aller voir Ōta Fine Arts, sa galerie officielle au Japon. Il m'a traité comme une vieille

18. Kusama Yayoi 草間弥生 (1929-?), artiste peintre et sculptrice japonaise, a acquis une véritable reconnaissance à la suite d'un séjour aux États-Unis (1957-1973) et reste aujourd'hui l'une des figures de proue du marché de l'art internationalisé. 
chaussette. « Alors, vous faites des trucs avec mon artiste sans m'en parler, et après vous voulez que je les achète ? Allez vous faire voir ». Finalement, Kusama m'a dit de les vendre où je voulais. [...] Moi, j'ai choisi des artistes que je savais pouvoir vendre. Le problème, c'est que ça fait des jaloux. Par exemple, la galerie Yamaguchi, à Osaka, n'était pas contente que j'aie exposé Koyama à l'ambassade. Elle voulait racheter les œuvres et refusait que je les montre à qui que ce soit avant. Mais moi, $\mathrm{j}$ 'avais monté ce projet dans une optique d'ensemble. Je leur ai dit : « Vous pouvez les racheter, mais moi je vais les montrer ». (Marchand de multiples)

Même s'il ne s'agit que de contrats oraux ou, le plus souvent, tacites, un galeriste n'est pas censé s'aventurer sur le terrain de ses confrères, autrement dit, vendre des artistes déjà soutenus ailleurs, à moins d'obtenir une permission très claire. Cette règle, strictement appliquée dans la capitale ou dans les quartiers regroupant de nombreux marchands, s'assouplit cependant à mesure que l'on s'éloigne sur le plan géographique. Ainsi les entretiens ont-ils montré que de nombreuses galeries tokyoïtes deviennent plus laxistes quand elles traitent avec des galeries régionales, en grande partie parce que la concurrence s'établit alors de manière moins frontale qu'à Tōkyō. Par exemple, alors qu'elles prendraient habituellement entre $5 \%$ et $10 \%$ de commission lors de la présentation d'un de leurs artistes à des partenaires potentiels (des « galeries suiveuses »), elles le font à titre gratuit quand il s'agit de marchands spatialement éloignés. C'est une manière d'élargir le réseau de diffusion, mais aussi de soutenir les maillons les plus faibles économiquement : sur le segment des artistes en voie de légitimation, les galeries régionales peinent le plus souvent à dégager des bénéfices, ce qui se répercute immanquablement sur les revenus des artistes, voire sur la viabilité à terme des expositions. Les efforts consentis par les galeries « principales » s'expliquent donc par une volonté de ne pas pénaliser leurs protégés. En cas de conflits d'intérêts, la galerie qui a soutenu l'artiste en premier a toujours la priorité.

Si des tensions peuvent surgir quant à la question du partage d'artistes déjà soutenus financièrement, concernant l'approvisionnement, les relations sont plutôt au soutien mutuel. 
292 Cahiers d'études japonaises n²2

Modes d'approvisionnement : une prise de risque en amont, compensée par des actions de solidarité

Où les galeries se fournissent-elles ? Dans l'ensemble, très en amont dans les circuits de reconnaissance, auprès des artistes débutants. Toutefois, le degré de prise de risque - particulièrement fort lorsqu'il s'agit de choisir au sein d'une offre pléthorique, dans une incertitude totale sur les chances de succès - varie selon leur type. Entre le tiers et la moitié des établissements à forte proportion de programmation fréquentent assidûment les expositions de fin d'étude et prennent très au sérieux la recommandation par un autre galeriste. À l'inverse, les galeries locatrices se trouvent davantage contactées par les artistes, qui voient dans leur participation financière un moyen de diminuer la probabilité des rejets. La découverte d'œuvres lors de visites de musées apparaît très minoritaire, ce qui laisse entrevoir un lien plus en amont entre galeristes et conservateurs. Enfin, si toutes les galeries recourent à la prise en dépôt, celles en programmation pure acquièrent davantage les œuvres avant de les vendre (28\% contre $19 \%$ ) et, surtout, sont les seules à pouvoir financer des projets en amont, à fréquenter les ventes aux enchères internationales et à proposer un salaire aux artistes.

Par ailleurs, il est un autre moyen efficace de s'approvisionner en œuvres : agir au sein des « sociétés d'échange » ${ }^{19}$. Le quart des galeries de l'échantillon participe ainsi à des syndicats ou des associations de marchands, où l'ancienneté, l'assise financière, le degré de programmation et la visibilité sont autant de modalités qui conditionnent l'intégration des membres. Ces lieux de rencontre constituent non seulement une source majeure d'approvisionnement sur le marché secondaire, mais permettent aussi de limiter les effets négatifs liés à l'incertitude sur la valeur des œuvres : de par les pratiques de solidarité qu'ils mettent en place, la possibilité est offerte à leurs membres de mutualiser les risques et d'agir au sein d'un cadre sûr, connu, cautionné par des pairs, qui contrôlent ensemble l'évolution des cotes. Ici, les galeries qui appartiennent à un syndicat (soit $25 \%$ de l'ensemble de la

19. Cléa Patin, « Le système des ventes d'œuvres d'art réservées aux professionnels au Japon », Ebisu, n ${ }^{\circ}$ 0, déc. 2013, p. 187-216. 
population enquêtée) participent à plusieurs d'entre eux, d'où le cumul de réseaux de sociabilité.

TAB. 5 : SYNDICATS ET ORGANISATIONS COLLECTIVES DES ENQUÊTÉS

\begin{tabular}{|c|c|c|}
\hline \multicolumn{2}{|c|}{ Syndicats et organisations collectives } & \multirow{2}{*}{$\begin{array}{c}\text { Galeries } \\
8 \%\end{array}$} \\
\hline $\begin{array}{l}\text { Syndicat des marchands } \\
\text { de peinture occidentale du } \\
\text { Japon }\end{array}$ & 日本洋画商協同組合 & \\
\hline $\begin{array}{l}\text { Syndicat des marchands de } \\
\text { gravures contemporaines } \\
\text { du Japon }\end{array}$ & 日本現代版画商協同組合（日版商） & $8 \%$ \\
\hline Tōkyō Art Club & 東京美術俱楽部 & $5 \%$ \\
\hline $\begin{array}{l}\text { Nouveau syndicat des } \\
\text { marchands d'art }\end{array}$ & 新美術商協同組合 & $5 \%$ \\
\hline $\begin{array}{c}\text { Syndicat des marchands } \\
\text { d'art de Tōkyō }\end{array}$ & 東京美術商協同組合 & $4 \%$ \\
\hline $\begin{array}{l}\text { Groupe d'entente mutuelle } \\
\text { des marchands de tout le } \\
\text { Japon }\end{array}$ & 全国美術商相互会（全美相） & $4 \%$ \\
\hline $\begin{array}{l}\text { Réunion intersyndicale de } \\
\text { marchands d'art }\end{array}$ & 協同組合美術商交友会（交友会） & $4 \%$ \\
\hline $\begin{array}{c}\text { Groupes Shinwa/Shinbi/ } \\
\text { Shinkō }\end{array}$ & 親和会/心美会/心交会 & $4 \%$ \\
\hline $\begin{array}{c}\text { Syndicat des marchands } \\
\text { d'estampes ukiyoe }\end{array}$ & 日本浮世絵商協同組合 & $2 \%$ \\
\hline $\begin{array}{c}\text { The International Fine } \\
\text { Print Dealers Association } \\
\text { (U.S.A.) }\end{array}$ & / & $2 \%$ \\
\hline $\begin{array}{c}\text { Syndicat du commerce et de } \\
\text { l'industrie }\end{array}$ & 商工組合 & $2 \%$ \\
\hline $\begin{array}{l}\text { Groupe d'entente mutuelle } \\
\text { des marchands d'Osaka }\end{array}$ & 大阪画商相互会 & $1 \%$ \\
\hline $\begin{array}{c}\text { Fédération des marchands } \\
\text { d'art du Kansai }\end{array}$ & 関西美術商連盟 & $1 \%$ \\
\hline
\end{tabular}


294 Cahiers d'études japonaises n²2

\begin{tabular}{|c|c|c|}
$\begin{array}{c}\text { Association des architectes } \\
\text { et artisans d'art du japon } \\
\text { Réseau des galeries } \\
\text { japonaises (G.N.A) } \\
\begin{array}{c}\text { Réunion des galeries d'art } \\
\text { contemporain }\end{array}\end{array}$ & 日本ギャラリー・ネットワーク協会 & $1 \%$ \\
$\begin{array}{c}\text { Groupe d'entente mutuelle } \\
\text { des marchands de Tōkyō }\end{array}$ & 東築美術工芸垷代美術画廊会議 & $1 \%$ \\
\hline $\begin{array}{c}\text { Nombre total de galeries } \\
\text { appartenant à des coopé- } \\
\text { ratives }\end{array}$ & 東京画商相互会 & $1 \%$ \\
\hline
\end{tabular}

En dehors des syndicats, les données de l'enquête montrent aussi que les galeries coopèrent activement entre elles : $40 \%$ organisent conjointement des expositions ; $24 \%$ publient ensemble des catalogues ; $22 \%$ s'allient pour lancer et promouvoir un artiste en particulier. Le but premier est de s'unir pour élargir le marché, en partageant au besoin un réseau de collectionneurs.

Autrefois, sous prétexte que je me situais sur le premier marché, je considérais que je ne devais vendre qu'à des collectionneurs privés. C'était une manière un peu naïve de penser... Plus je prends de l'expérience, plus je réalise à quel point la priorité pour une galerie est d'aider ses artistes à se faire une réputation. Or, si plusieurs galeries se concentrent sur un même artiste, son marché s'élargit. Au fond, il faut être au moins deux marchands pour accroître une clientèle. Moi, j'avais toujours accordé plus d'importance au soutien des artistes qu'à l'aspect commercial, à la rentabilité. Je pensais qu'il suffisait de se donner à fond pour les artistes, pendant cinq ou dix ans, en leur permettant d'organiser d'excellentes expositions... Mais après vingt ans d'activité, je vois que cela ne leur a pas forcément rendu service. Il vaut mieux pour eux d'exposer dans un grand nombre de lieux différents. Les galeries, en unissant leurs forces, peuvent partager leur réseau de collectionneurs. (Galerie Soh) 
Résultat des relations d'interdépendance sur le marché domestique dont dépend la survie de nombreuses galeries, le degré d'internationalisation de l'échantillon apparaît en revanche faible.

\section{$\underline{\text { Un faible degré d'internationalisation }}$}

Même les galeries japonaises actives dans l'art actuel accueillent paradoxalement peu d'artistes étrangers : seulement $2 \%$ invitent des artistes asiatiques et 10,3\% des artistes occidentaux, dans une proportion supérieure à $30 \%$. La vente à des galeries étrangères et l'achat dans des ventes aux enchères internationales jouent également dans des proportions restreintes, puisque plus des deux tiers ne mènent pas ce genre d'activité.

Les galeries japonaises accueillent extrêmement peu d'artistes étrangers. Elles sont très fermées, ce qui pose un vrai problème. De plus, leur taille est très modeste. La triennale de Yokohama n'attire personne ! Même les participants ne se déplacent pas. Ils se contentent d'envoyer leurs œuvres. Pour eux, ce n'est pas rentable, vu le manque d'achat muséal, d'expositions d'art contemporain et d'artistes internationaux dans les galeries. Le Japon, c'était dynamique jusque dans les années 1980. Ensuite la bulle est apparue, il y a eu emballement... puis arrêt total. Depuis, les professionnels du monde de l'art ne viennent pas. (Artiste fortement internationalisé L)

Pourtant, toutes ont conscience de l'importance de la participation à des foires internationales. $33 \%$ y sont représentées, tandis que beaucoup cherchent à nouer des liens avec des artistes locaux dans d'autres villes d'Asie (Shanghaï, Canton, Hong Kong, Singapour). Même les plus modestes affirment vouloir débaucher des artistes en Corée et en Chine du fait de leur proximité géographique. Cela démontre l'existence d'un idéal d'internationalisation, même s'il peine à se concrétiser, qui se couple à la volonté d'augmenter le nombre des acheteurs. 
296 Cahiers d'études japonaises n²2

\section{À la rencontre de la clientèle}

\section{Dépendance à l'égard d'une poignée de collectionneurs}

Tous les galeristes rencontrés déclarent faire face à « une demande très restreinte, mais passionnée ». Qui donc sont ces acheteurs ? Dans l'enquête, les collectionneurs individuels constituent de loin la première catégorie de clients : ils représentent la principale source de revenus pour $53 \%$ des galeries. Or, malgré la volonté affichée de « diffuser la culture dans toutes les couches de la société », une galerie ne s'adresse pas à un consommateur lambda. Il s'agit majoritairement de personnes de sexe masculin, entre 40 et 60 ans, fonctionnaires, cadres ou chefs d'entreprises, avec un niveau d'éducation élevé. Tous ne sont cependant pas des « héritiers » : certains partagent des origines modestes, l'accès à un haut niveau de culture constituant une sorte de revanche sur le plan social.

Ici, contrairement à nos attentes, sur les enquêtés ayant répondu dans quelle mesure ils dépendent de leurs cinq clients les plus importants (56\%), $37 \%$ affirment avoir une dépendance très faible, tandis que $12 \%$ avancent une dépendance très forte (cf. graph. 5). Ils parviennent donc à diversifier efficacement leurs sources de revenus et à minimiser les risques. Cependant, $3 \%$ reconnaissent aussi s'en remettre entièrement à ces cinq clients principaux, auquel cas la disparition de l'un d'entre eux menacerait de se répercuter gravement sur leur trésorerie. Toutefois, cela ne devient véritablement une forme de fragilité que si ces galeristes dépendent complètement d'un seul client (auquel cas il n'y a aucune diversification du risque). 


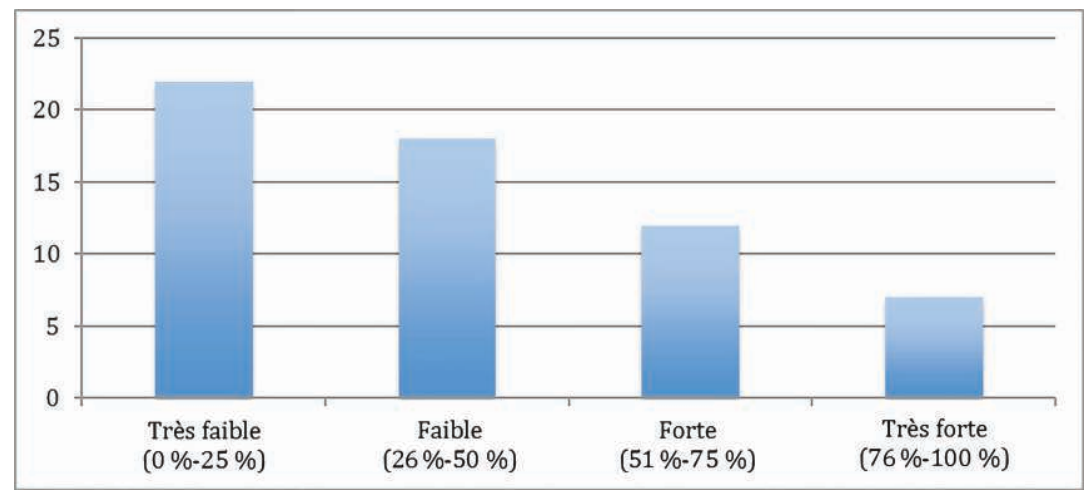

\section{GRAPH. 5 : PART DES CINQ CLIENTS LES PLUS IMPORTANTS DANS LE CHIFFRE D'AFFAIRES \\ Source : Cléa Patin.}

Bien qu'il leur en coûte - nombreux sont ceux qui insistent sur le fait que les prix sont déjà très bas et que les rabais instaurent une forme d'injustice entre les acheteurs - les galeristes sont souvent obligés de négocier et de consentir aux clients des facilités de paiement. Toutefois, une perte trop forte de leur côté se répercute irrémédiablement sur le revenu des artistes. À cela s'ajoute le problème des « mauvais payeurs ».

Si seulement les gens pouvaient payer rapidement quand ils acquièrent des œuvres... Mais non, la plupart s'acquittent en versant des mensualités. Que les clients soient de simples employés, des travailleurs indépendants ou des personnes fortunées, que cet argent provienne du rendement de titres, du rapport d'intérêts ou d'un surplus retiré de la vente de terrains, très peu de personnes payent rubis sur ongle... Et certains ne s'acquittent pas de leurs dettes. Parce que les ouvres ne constituent pas des produits de première nécessité. (Galerie Shinobazu)

Ces soucis mis à part, la relation qui se noue entre amateurs d'art et galeristes, constructive, dynamique et sans cesse réactivée, forme les bases d'un cheminement 
commun pour choyer une collection, dont chacun retire de la fierté. La sélection s'opère alors des deux côtés : le collectionneur choisit son marchand avec soin, de même qu'un bon galeriste s'attache un petit nombre de clients, qui continuent de le soutenir dans les creux de la conjoncture.

Je vends les meilleures œuvres à mes clients préférés. À ceux qui achètent uniquement par vanité ou orgueil, je vends des œuvres différentes. Je fais le tri. (Galerie NCA 日動コンテンポラリー アート)

Je préfère prendre mon temps pour attraper un million de yens plutôt que la pièce qui se trouve sous mon nez. Quand on vend à des clients, il ne s'agit pas uniquement de business. On ne vend pas à n'importe qui, sous prétexte que cela rapporterait de l'argent. Moi, je suis un sale type, alors je ne vends qu'aux clients qui me plaisent. Je ne choisis que ceux qui seront capable de choyer les œuvres que je leur propose. Parce qu'elles sont un peu mes enfants. On collectionne par amour. (Galerie Kotōken)

Le rôle des marchands dans la formation du goût est d'autant plus déterminant que les acheteurs sont producteurs de leur propre utilité, à savoir la sensation de plaisir associée à la consommation d'un bien : les biens d'art présentent une fonctionnalité limitée, mais requièrent une participation intellectuelle et émotionnelle très forte de la part du consommateur, qui produit sa propre satisfaction à partir des caractéristiques objectives de l'œuvre, autant que de ses propres compétences, cognitives ou affectives ${ }^{20}$.

Si les collectionneurs privés forment le socle principal des acheteurs, où se situent les entreprises ? Celles-ci se placent dans la clientèle de l'échantillon au même niveau que les musées et les galeries étrangères, après les sociétés d'échanges

20. George J. Stigler, Gary S. Becker, “De gustibus non est disputandum”, American Economic Review, no 67, 1977, pp. 76-90. C'est aussi à ce titre que la formation du goût s'avère sujette à un fort déterminisme social. Pierre Bourdieu, Alain DARbel, L'Amour de l'art: les musées européens et leur public, Paris, Les Éditions de Minuit, 1969. 
et les grands magasins. Force est de constater que l'implication de ces dernières a considérablement diminué après l'éclatement de la bulle spéculative : jusque dans les années 1980, les fondateurs des grands groupes japonais (kigyō ōnā 企 業オーナー) avaient suffisamment de poids et d'autorité pour imposer leurs choix lorsqu' ils voulaient constituer une collection, mais à partir de la « décennie perdue », leurs successeurs, pour la plupart des anciens salariés (salaryman shachō サラリーマン社長), n’ont plus bénéficié de cette marge de manœuvre, sous la pression accrue tant des actionnaires que des employés. Désormais, si collection d'entreprise il y a, c'est plutôt au sein de petites ventures très dynamiques, entièrement sous l'emprise de la direction.

Les entreprises ne nous achètent plus rien. Pendant la bulle, les banques, les grands groupes acquéraient des œuvres d'art d'artistes vivants pour les offrir à leurs clients... Mais aujourd'hui, rien. (Galerie Nichidō)

Les entreprises dépensent de moins en moins dans le domaine de la culture. Leurs nouveaux bâtiments, tout en verre, ne sont pas conçus pour accueillir des tableaux. Certes, elles ont toujours préféré augmenter leurs fonds propres plutôt que d'investir dans l'art, mais autrefois, certains directeurs d'entreprises soutenaient les musées, faisaient don de leurs collections aux collectivités locales, ou encore mettaient à disposition une partie de leurs locaux [pour organiser des événements culturels]. Ces PDG deviennent rares. Aujourd'hui, si les fondateurs d'entreprises ont encore une certaine liberté d'action, les directeurs dont la carrière repose sur une promotion progressive au sein de l'entreprise craignent trop de s'attirer les foudres des actionnaires. (Galerie Shinobazu)

\section{Des modes de diffusion plutôt timides}

La quasi-totalité des galeries ont recours à des envois postaux, qui semblent fournir le meilleur retour sur investissement. La moitié annonce leurs expositions par e-mail, même si ce mode de promotion présente le risque d'agacer des personnes 
300 Cahiers d'études japonaises n²2

déjà surchargées de courriels et de spams. Le tiers publie des catalogues, au nombre d'un ou deux par an, avec des tirages autour de 900 exemplaires. Enfin, un peu plus du quart impriment des brochures et des prospectus, déposés dans des galeries proches, via un système réciproque d'échange de publicité et d'information.

Les galeries s'appuient sur des listes de collectionneurs potentiels, élaborées au fil des visites et des ventes antérieures, qui contiennent entre 600 et 3000 noms, selon leur taille et leur réputation. Pourtant, quelques collectionneurs seulement se déplacent et un nombre plus restreint encore passe le cap de l'achat. Ainsi, la plupart des petites galeries d'art contemporain s'estiment satisfaites quand elles parviennent à fidéliser une vingtaine d'acheteurs réguliers.

Par ailleurs, si l'information fuse pour les habitués du marché, le premier pas dans le monde des marchands d'art est difficile à effectuer. En effet, très peu d'information filtre en dehors du milieu. Par exemple, si les galeries épinglent dans leur entrée des informations relatives aux expositions du quartier, encore faut-il y pénétrer une première fois. De même, l'information relative aux événements annuels (Ginza night, Roppongi night, etc.), dont le but affiché est d'attirer un large public (ouverture exceptionnelle jusqu'à $22 \mathrm{~h}$, éclairage des œuvres aux flambeaux, etc.) n'est pas mentionnée dans les grands quotidiens. Ainsi, si les galeries affirment faire des efforts colossaux en matière de communication, elles ne sortent au fond pas vraiment de leur propre monde.

De nombreuses personnes seraient prêtes à acheter une œuvre, mais les galeries ne font aucun effort. Ou plutôt, elles en font, mais de manière inefficace. Par exemple, la brochure sur la Ginza night, où était-elle ? Dans les galeries. Donc accessible qu'à ceux qui ont l'habitude d'y mettre les pieds. Si elle était parvenue aux OL et aux salary men, elle aurait attiré au moins cinquante personnes! Pareil pour l'événement prévu en juillet, au cours duquel les galeries se regroupent pour organiser des expositions en commun. Une carte a été imprimée... Mais où la trouve-t-on ? Dans le milieu. Les gens ne viennent pas parce qu'ils ne savent pas. On me ressasse sans cesse que les informations sont dans des magazines spécialisés ou sur Internet. Mais est-ce que les employés normaux lisent ce genre de rubrique ? Il vaudrait mieux poster une annonce de temps en 
temps dans les grands journaux. Par ailleurs, on peut toujours mettre autant de tableaux qu'on veut sur Internet, personne ne va les chercher. Pareil pour les mails, qui ne sont pas lus. Les galeristes et les artistes ont beau dire « on a mis plein d'informations! », elle ne touche que ceux qui ont déjà un intérêt pour l'art. Et ils croient faire des efforts... (Collectionneur Y)

Même si toutes les galeries se situent au cœur des réseaux qui relient artistes et collectionneurs, l'efficacité de leur action se révèle en définitive très inégale selon leurs profils sociologiques.

\section{Hiérarchie et mécanismes de prédation dans la construction de la valeur économique des œuvres}

\section{«Une galerie qui ne vend pas est une manvaise galerie »}

L'enjeu de la relation artiste-marchand concerne fondamentalement la construction de la valeur économique des œuvres, à l'intersection des institutions culturelles et du marché21. De la capacité ou non à endosser cette responsabilité dépend, aux yeux des artistes, le fait d'être considéré comme une « bonne galerie », ou même une galerie à part entière.

Exemple très pur de la manière dont cette valeur est construite, citons les débuts d'un jeune plasticien, qui exposait en avril 2007 au sein d'une association de collectionneurs (AIT). Encore étudiant en $4^{e}$ année à l'École des BeauxArts de Musashino, ses prix ne se fixaient alors modestement qu'à 5000 yens le point. À l'entendre, l'université ne fournissait aucune information pratique sur la manière de s'insérer sur le marché de l'art contemporain, l'obligeant à trouver des solutions par lui-même. Il disait faire face à une grande solitude, voire au mépris de ses professeurs et à la jalousie de ses camarades (ce discours mérite d'autant plus d'être relativisé qu'il a reçu l'année suivante un prix de son université).

21. Raymond Moulin, op. cit., 1992 ; Nathalie Moureau, Analyse économique de la valeur des biens d'art, Paris, Economica, 2000. 
Depuis, il a été récompensé par l'《Art award Tōkyō アートアワードトーキ $\exists ー 2008$ », a été sélectionné plusieurs fois au salon des jeunes artistes « Tōkyō wonder wall トーキョーワンダーウォール », a participé à la foire GEISAI de Murakami Takashi et a été distingué par la galerie allemande Strenger. Un collectionneur japonais rencontré à GEISAI, qui réside à New York, l'a même introduit dans une petite galerie de Chelsea. On pourrait croire à un coup de chance... Pourtant, le témoignage de la galeriste à l'origine de l'exposition de 2007 montre que ses débuts ont été savamment orchestrés :

Il n'est pas encore connu, mais a beaucoup d'ambition. Il veut devenir incontournable, comme Nara Yoshitomo ou Murakami Takashi, et s'attirer les faveurs des grandes galeries commerciales (Taki Kenji, Yamamoto Gendai ou Koyama Tomio). Bref, il cherche à devenir riche et célèbre, ici et maintenant, sans pour autant faire des compromis sur ses œuvres. Pendant l'exposition d'AIT, il a beaucoup vendu. Des marchands autres que sa galerie principale se sont déplacés, comme NCA. J'ai alors réalisé à quel point l'enjeu véritable est de construire le marché. Au fond, peu importe que l'œuvre soit bonne ou mauvaise, ou que ces marchands l'apprécient ou non. La vraie question réside dans le fait de créer un marché et de le maintenir. Pour préserver les cotes, ces marchands organisent des campagnes publicitaires. Une autre méthode consiste à porter une œuvre aux enchères, tout en demandant à une connaissance de procéder à l'achat pour, mettons, dix millions de yens. Ce prix se trouve alors étiqueté sur l'artiste et devient la valeur de référence. Avec ces techniques, ils peuvent habilement maintenir et contrôler le marché. Il s'agit de commerce pur. J'ai compris à quel point j'avais été naïve de vouloir tout bonnement diffuser au plus grand nombre les œuvres qui me plaisaient (c'est la raison pour laquelle j'ai fait faillite !). Je jouais à la dînette. Or, du point de vue d'un artiste ambitieux, une galerie qui ne vend pas est une mauvaise galerie, une galerie inutile, une galerie avec laquelle on ne peut s'entendre. (Ex-Galerie Isogaya ギャラリーいそがや) 
Pour ce qui est de la capacité à imposer un artiste, toutes les galeries ne jouent pas à égalité. Au contraire, l'analyse factorielle distingue cinq groupes précis, dont l'efficacité varie considérablement.

Les enseignements de l'analyse factorielle : une classification en cinq branches hiérarchisées

Toutes les galeries de l'échantillon peuvent être classées selon leur positionnement sur le marché. L'axe 1 indique le degré de performance, tandis que l'axe 2 mesure leur engagement.

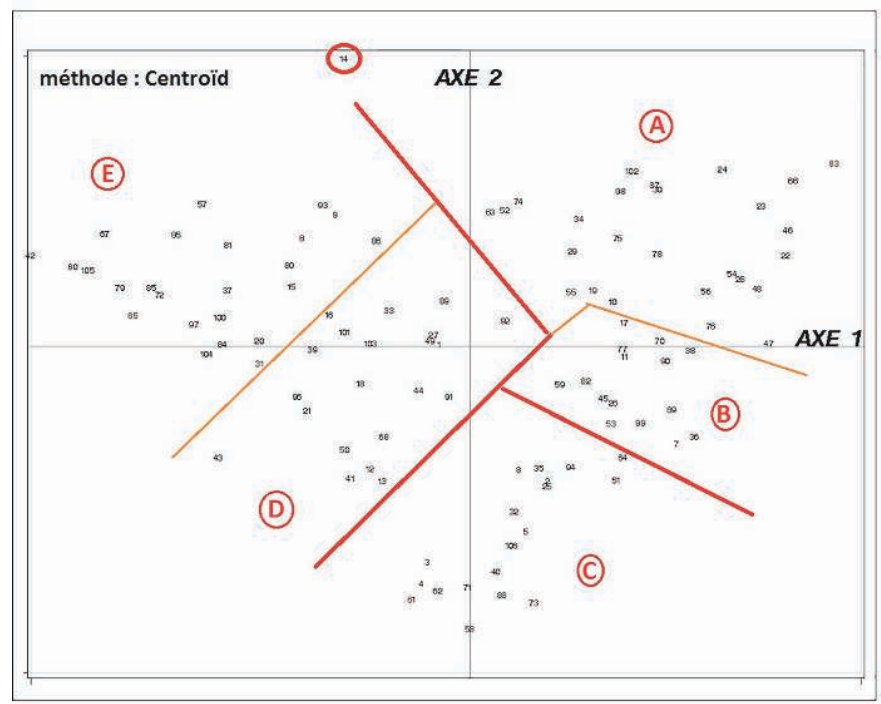

GRAPH. 6 : CLASSIFICATION HIÉRARCHIQUE ASCENDANTE PAR LA MÉTHODE CENTROÏD

Source : Cléa Patin. Constitué à partir des résultats de l'enquête sur 106 galeries tokyoïtes (les nombres renvoient au code chiffré de chacune d'entre elles) 
Ici, deux mécanismes de compétition émergent : le premier, vertical, joue au niveau de la taille, de la puissance financière et de la réputation; le second, horizontal, concerne la segmentation du marché selon les catégories de biens ou les styles. Nous pouvons en outre observer une solution de continuité par ressemblance ( $\mathrm{AB} B C \mathrm{CD} \mathrm{DE})$, sur plusieurs variables : la surface, le nombre d'employés, la programmation, le chiffre d'affaire, l'importance des clients principaux, mais aussi l'ancienneté et la valeur des œuvres. Les A sont les majors. Présentes à la fois sur le premier et le second marché, elles bénéficient d'une implantation ancienne et solide. Suivent les B, galeries d'ouverture, fortement implantées sur le marché international. Il s'agit ici de la frange ayant réussi dans le domaine de l'art contemporain qui, avec le temps, pourra prétendre au statut des A. Elles ont tout intérêt à laisser subsister en dessous d'elles des galeries plus modestes, chargées de la découverte et du lancement des artistes, quitte à user de leur nom pour débaucher les artistes les plus prometteurs. Cette génération de galeries innovantes, mais à faibles moyens financiers, est caractérisée par les C. Récentes, éclectiques, elles jouent un rôle primordial pour les artistes en début de carrière. Derrière elles viennent enfin les galeries pour lesquelles la location permet d'équilibrer les comptes. Certaines présentent des atouts sur le plan patrimonial, qu'il faut faire fructifier (les D), d'autres surnagent en faisant simplement porter aux artistes la majeure partie des coûts d'exposition (les E).

\section{Le renforcement d'une structure sous forme d'oligopole à franges : vers une fragilisation à terme}

Cette hiérarchie des galeries démontre une fois de plus que le milieu se structure en grappes ${ }^{22}$. En son sein, les parcours ne sont pas statiques : les artistes se trouvent happés au fil de leur carrière d'un échelon à l'autre, chaque étape apportant son lot d'avantages - nouveaux financements pour leur création, réseau de collectionneurs étendu, reconnaissance par les experts.

Mais si ce système de progression dans le lancement et la reconnaissance des artistes est bien connu, l'analyse factorielle met ici en lumière un impact encore

22. Marcia N. Bystryn, op. cit., 1981 ; Katherine GiUffre, op. cit., 1991. 
inégalé de la force financière comme arme de production des artistes - phénomène qui semble nouveau au Japon par son ampleur. C'est désormais elle qui permet de mobiliser l'ensemble des acteurs du marché, au risque d'exacerber des mécanismes de prédation, qui pourraient fragiliser à terme l'écosystème des galeries et créer de nouveaux déséquilibres.

De fait, le marché prend de plus en plus la forme d'un oligopole à frange. Quelques grosses galeries maîtrisent l'essentiel du marché, tandis qu’à leur périphérie gravitent une myriade de PME (galeries ou collectifs d'artistes) « amenées à satisfaire des demandes de faibles volume ou des demandes spécifiques $\gg^{23}$. Le mécanisme est le suivant : les firmes du cœur laissent à celles en marge de l'oligopole le soin de découvrir et de soutenir les nouvelles tendances, sans subir les coûts de promotion ni les risques liés aux échecs potentiels, puisqu'elles peuvent plus tard les déposséder de leur travail, en contrôlant leur capital ou en séduisant leurs artistes les mieux reconnus. À l'inverse, les firmes périphériques « fixent leurs prix sous l'ombrelle des firmes dominantes et tendent à assumer la majeure partie de l'innovation $»^{24}$. Quand bien même le suivi d'artistes à tous les stades de carrière s'avère indispensable pour assurer leur survie (elles gèrent un « portefeuille d'artistes »), à travers la réinjection du fruit des investissements réussis sur la jeune génération, elles se trouvent soumises à un effet de prédation des artistes les plus profitables, ce qui les prive d'un précieux retour sur investissement. Ainsi, autant le cœur bénéficie d'un pouvoir de marché qui lui assure une pérennité sur le long terme - même si la mondialisation tend à accentuer la concurrence des ténors à l'échelle internationale - autant les firmes de la frange vivent une existence instable et précaire.

Jusqu' ici, les galeries se spécialisaient chacune dans un domaine. Certaines s'occupaient de soutenir les jeunes talents fraîchement sortis des Beaux-Arts. Une fois qu'un de leurs poulains sortait $\mathrm{du}$ lot, elle se faisait contacter par une galerie plus puissante qui prenait le relais. Et ainsi de suite jusqu'au sommet de la pyramide,

23. Françoise Benhamou, Nathalie Moureau, Dominique Sagot-Duvauroux, op. cit., 2001, p. 126.

24. Ibid. 
constitué des majors. Mais il y avait des règles, des gardes fous. En tout état de cause, ça passait par des marchands. Aujourd'hui, c'est la loi de la jungle. Des majors sont venus débaucher directement des jeunes créateurs dans cet immeuble même, juste sous le nez de leurs galeristes. Et pas seulement ici! Les galeries semi-programmatrices se donnent beaucoup de mal pour donner leur chance aux débutants et se les font voler. Il suffit pour un galeriste célèbre de tirer un jeune par la manche et de lui dire « ça te dirait d'exposer chez moi ? ». S'il avait l'intention de le suivre sur le long terme, pourquoi pas, mais ce n'est pas le cas. L'artiste, tout juste sorti de l'école, accepte avec empressement. S'il émet quelques réserves, le galeriste lui réplique, condescendant : «Très bien, reste dans ta petite galerie ; ils ne risquent pas de t'acheter une œuvre majeure ». Pour l'appâter, on lui propose d'acheter une centaine de points. Et marché conclu. Ensuite, le galeriste répète son manège dans d'autres galeries. Puis il réunit tous ces artistes pour organiser une exposition collective dans ses propres locaux. Si l'un d'eux perce, tant mieux. Sinon, out, bye-bye ! Les artistes sont jetés. Le problème, c'est que le jeune ne peut plus revenir dans son ancienne petite galerie : il est grillé. C'est arrivé maintes fois ! Il n'y a plus de règles. On est passé à la chasse aux idoles. (Collectionneur Y)

De tels mouvements de concentration ne peuvent que favoriser les tendances spéculatives (les galeries dotées de capitaux importants imposent artificiellement leurs artistes dans les ventes aux enchères) ou les collusions entre firmes dominantes, qui abusent de leur pouvoir de marché. Enfin, comme le soulignent Françoise Benhamou, Nathalie Moureau et Dominique Sagot-Duvauroux, « entre l'arrivée des maisons de ventes aux enchères, à la limite du premier marché, et un réseau de soutien à la création contemporaine qui se prête souvent mal à une exploitation commerciale, les petites galeries se trouvent de plus en plus prises en 
étau ${ }^{25} \gg$. Or, contrairement au monde de la musique, elles s'avèrent sans recours en cas de litige, du fait de la quasi-absence de contrats écrits.

Acteurs centraux du marché de l'art puisqu'elles se situent au cœur de l'intermédiation entre l'offre et la demande, les galeries influencent au plus haut point la formation de la valeur économique des œuvres, tant sur le marché secondaire (notamment au sein des réunions d'échange) que sur le marché primaire. Or, contrairement aux maisons de vente aux enchères, elles endossent la majeure partie des risques. Chargées de sélectionner les artistes et de leur donner les moyens de faire carrière dans un contexte de surproduction de l'offre et d'incertitude radicale sur la valeur future des biens, elles se voient obligées de développer plusieurs méthodes susceptibles de viabiliser leur activité : se polariser par quartiers, augmenter ou diminuer le degré de location en fonction des déficits, agir simultanément sur le premier et le second marché, ou encore s'unir pour mener des actions en commun (approvisionnement au sein des syndicats, publication de catalogues, partage de la représentation d'un artiste, organisation conjointe d'expositions, etc.). Si ces actions leur permettent de surnager, elles limitent aussi leur capacité à rayonner à l'international et ne les protège pas de l'exacerbation d'une hiérarchie entre elles : l'absence de contrat, qui se cumule avec une structuration sous la forme d'un oligopole à frange, accentue la vulnérabilité des plus modestes, qui sont pourtant à la base des soutiens octroyés aux artistes en voie de légitimation et qui se dédient à l'innovation artistique. Pour survivre, certaines devront sans doute faire des compromis sur leur degré de programmation/location, en acceptant de louer leurs locaux à des artistes de passage. À l'inverse, les plus fortes financièrement, conscientes de l'adage sur le marché de l'art « à fort risque, fort retour sur investissement », au sens des gains comme des pertes, participeront au renforcement d'un star system à la japonaise, où une poignée de vedettes reconnues sur la scène internationale raflera la majorité des revenus et fera miroiter à toute une génération d'artistes débutants les atouts d'une carrière « express », qui permet certes des réussites exemplaires, mais décuple aussi la probabilité de l'échec.

25. Id., p. 104. 
308 Cahiers d'études japonaises n²2

\section{Bibliographie indicative}

\section{Travaux}

Benhamou Françoise, Moureau Nathalie, Sago-Duvauroux Dominique, Les Galeries d'art contemporain en France. Portraits et enjeux dans un marché mondialisé, Paris, La Documentation française, 2001, 220 p.

Bourdieu Pierre, Darbel Alain, L'Amour de l'art: les musées et leurpublic, Paris, Les Éditions de Minuit, 1969, 256 p.

Bystryn Marcia N., "Variation in Artistic Circles", The Sociological 2uarterly, vol. 22, nº 1, 1981, pp. 119-132.

Bystryn Marcia N., "Art Galleries as Gatekeepers: The Case of the Abstract Expressionists”, Social Research, vol. 45, 1978, pp. 390-408.

Guiffre Katherine, "Sandpiles of opportunity: Success in the Art World", Social Forces, vol. 77, n 3, 1999, pp. 816-817.

Lucken Michael, L'Art du Japon au vingtième siècle, Paris, Hermann, 2001, 350 p.

Menger Pierre-Michel, « L’hégémonie parisienne. Économie et politique de la gravitation artistique », Annales E.S.C., « Mondes de l'art », nov/déc. 1993, p. $1565-1600$.

Menger Pierre-Michel, « Les artistes en quantités. Ce que sociologues et économistes s'apprennent sur le travail et les professions artistiques », Revue d'économie politique, vol. 120, janv. 2010, p. 205-236.

Menger Pierre-Michel, Le Travail créateur : s'accomplir dans l'incertain, Paris, Gallimard-Seuil, 2009, 672 p. 
Moulin Raymonde, Le Marché de la peinture en France, Paris, Les Éditions de Minuit, 1967, 624 p.

Moulin Raymonde, L'Artiste, l'institution et le marché, Paris, Champs Flammarion, 1992, 437 p.

Moulin Raymonde, Le Marché de l'art : mondialisation et nouvelles technologies, Paris, Flammarion, 2003, 154 p.

Moureau Nathalie, Analyse économique de la valeur des biens d'art, Paris, Economica, 2000, 360 p.

NiHON YōGASHŌ KYōDō KUMIAI 日本洋画商協同組合, Nihon yōgashō-shi 日本洋画商史 (Histoire des marchands d'art occidental), Tōkyō, Bijutsu shuppan-sha 美術出版社, 1985, 560 p.

O'Flaherty Brendan, City Economics, Massachusetts, Harvard University Press, $2005,587 \mathrm{p}$.

Patin Cléa, La Fabrique de l'art au Japon : Portrait sociologique d'un marché de l'art, Coll. «Réseau Asie », CNRS éditions, 2016,391 p.

Patin Cléa, « Le système des ventes d'œuvres d'art réservées aux professionnels au Japon (kōkankai) », Ebisu, no 50, 2013, p. 187-216.

SEGI Shin.ichi 瀬木慎一, «Bijutsu shijō rēdā » 美術市場レーダー (Le radar du marché de l'art), Shin bijutsu shinbun 新美術新聞 (Nouveau journal des arts), 21 juillet 2001 et 21 août 2008.

SEGIShin.ichi, Kokusai/Nihon bijutsu shijōsōkan : baburukara defure e 1990-2009 国際/日本美術市場総監：バブルからデフレへ 1990-2009 (Panorama général du marché de l'art international et japonais de la bulle à la déflation 1990-2009), Tōkyō, Fujiwara shoten 藤原書店, 2010, 618 p. 


\section{CIPANGO}

310 Cahiers d'études japonaises n²2

Stigler George J., Becker Gary S., "De gustibus non est disputandum", American Economic Review, n ${ }^{\circ} 67,1977$, p. 76-90.

TŌKYŌ BIJUTSU KURABU HYAKUNEN-SHI HENSAN IIN-KAI 東京美術俱楽部 百年史編纂委員会, Bijutsushōno hyakunen: Tökyō bijutsu kurabu byakunen$s h i$ 美術商の百年一東京美術俱楽部百年史 (Le siècle des marchands d'art : 100 ans d'histoire du Club des Arts de Tōkyō), Tōkyō, Tōkyō Bijutsu kurabu/Tōkyō bijutsu-shō kyōdō kumiai 東京美術俱楽部・東京美術商 協同組合, 2006, 1472 p.

TŌKYŌ BIJUTSU KURABU 東京美術俱楽部, Tökyō bijutsu shijō-shi 東京美 術市場史 (Histoire du marché de l'art tokyoïte), Tōkyō, Tōkyō bijutsu kurabu, 1979, 956 p.

\section{Annuaires d'art et guides des galeries}

Gekkan gyararī 月刊ギャラリー, Gyararī sutēshon ギャラリーステーショ ソ, Tōkyō, mars-mai 2008.

Bijutsu nenkan 美術年鑑, Tōkyō, Bijutsu nenkan-sha 美術年鑑社, 1987, 1992, 1997, 2002, 2007.

Bijutsu techō nenkan 美術手帳年鑑, Tōkyō, Bijutsu shuppan-sha 美術出版社, 2007.

\section{Conférence}

HATAKEyAma Kōji 畠山耕治, 《Bijutsu sakka no kakeibo 》美術作家の家計 簿 (Carnet de dépenses d'un artiste), conférence organisée le 23 mars 2007 par Art Interactive Tōkyō アート・インタラクティヴ東京 dans le cadre 
du cycle Bijutsu korekutā no gimon ni kotaete 美術コレクターの疑問に答 えて (Réponses aux questions des collectionneurs).

Résumé: Au Japon comme en France, les galeristes jouent un rôle irremplaçable au cœur du marché de l'art. En effet, ils se chargent d'exhumer les nouvelles tendances artistiques - auquel cas ils opèrent dans un contexte d'incertitude radicale à partir d'un excès structurel d'offre - et se mettent en rapport avec des collectionneurs, des musées, des acheteurs, tout en entrant souvent en concurrence avec des maisons de vente aux enchères. Par la formation de la demande et la sélection au sein de l'offre, ils contrôlent une partie du mécanisme de construction des valeurs artistiques. À partir d'une enquête de terrain, par entretien et par questionnaire, nous explorerons ici la manière dont les galeristes japonais s'efforcent de relever les défis qu'implique ce rôle, à travers une analyse de leur place globale sur le marché de l'art (structure, revenus, répartition spatiale, démographie, etc.), puis une description de leur fonctionnement au quotidien (relation aux artistes, fidélisation de la clientèle, etc.), dans la ville de Tōkyō, avant d'évaluer les risques de fragilisation qui les menacent à terme, du fait de mécanismes de prédation exacerbés.

Abstract: In Japan, as well as in France, gallery owners play an essential role, central within the art market. Indeed, they are responsible for discovering new artistic trends, an operation which requires them to maneuver in a context of radical uncertainty, by drawing from a pool of artists in structural excess. All the while, they also build strong relationships with collectors, museums, buyers, often competing with auction houses. Since they strongly contribute to forming the demand and carrying out a selection within the supply, they control a major part of the mechanisms of construction of artistic values. Building on the results of our fieldwork survey, during which we conducted numerous interviews and compiled the data obtained via our questionnaire, we explore how Japanese gallery owners strive to meet the challenges of their role, by analyzing their global status in the art market (structure, income, spatial distribution, demography, etc.), and understanding their daily operation (relationships with artists, creation of customers loyalty, etc.), with a special focus on Tökyō. Finally, we evaluate the risks of the profession being progressively weakened, due to exacerbated predation mechanisms among them. 


\section{CIPANGO}

312 Cahiers d'études japonaises n²2

Mots-clés : galeries japonaises, galeriste, marché de l'art, valeur artistique, enquête de terrain, marchand d'art, analyse factorielle, recensement

Keywords: Japanese galleries, gallerist, art market, art dealer, artistic value, field study, artistic value, inventory, factor analysis

キーワード: 美術市場 日本の画廊 美術商 ギャラリストフィールド ワーク一覧表 因子分析 